\title{
Search for a Supersymmetric Partner to the Top Quark in Final States with Jets and Missing Transverse Momentum at $\sqrt{s}=7 \mathrm{TeV}$ with the ATLAS Detector
}

\author{
G. Aad et al.* \\ (ATLAS Collaboration)
}

(Received 7 August 2012; published 20 November 2012)

\begin{abstract}
A search for direct pair production of supersymmetric top squarks $\left(\tilde{t}_{1}\right)$ is presented, assuming the $\tilde{t}_{1}$ decays into a top quark and the lightest supersymmetric particle, $\tilde{\chi}_{1}^{0}$, and that both top quarks decay to purely hadronic final states. A total of 16 (4) events are observed compared to a predicted standard model background of $13.5_{-3.6}^{+3.7}\left(4.4_{-1.3}^{+1.7}\right)$ events in two signal regions based on $\int \mathcal{L} d t=4.7 \mathrm{fb}^{-1}$ of $p p$ collision data taken at $\sqrt{s}=7 \mathrm{TeV}$ with the ATLAS detector at the LHC. An exclusion region in the $\tilde{t}_{1}$ versus $\tilde{\chi}_{1}^{0}$ mass plane is evaluated: $370<m_{\tilde{t}_{1}}<465 \mathrm{GeV}$ is excluded for $m_{\tilde{\chi}_{1}^{0}} \sim 0 \mathrm{GeV}$ while $m_{\tilde{t}_{1}}=445 \mathrm{GeV}$ is excluded for $m_{\tilde{\chi}_{1}^{0}} \leq 50 \mathrm{GeV}$.
\end{abstract}

DOI: 10.1103/PhysRevLett.109.211802

PACS numbers: 12.60.Jv, 13.85.Rm, 14.80.Ly

The standard model (SM) is a successful but incomplete theory of particle interactions. Supersymmetry (SUSY) [1-9] provides an elegant cancellation of the quadratic mass divergences that would accompany a SM Higgs boson by introducing supersymmetric partners of all SM particles, such as a scalar partner of the top quark $(\tilde{t})$. Like $t \bar{t}$, direct $\tilde{t} \tilde{t}^{*}$ is produced primarily through gluon fusion at the Large Hadron Collider (LHC). The production cross section depends mostly on the mass of the top partner and has minimal dependence on other SUSY parameters [10-12]. The LHC enables searches for direct $\tilde{t} \tilde{t}^{*}$ production at higher mass scales than previous accelerators [13-27]. The viability of SUSY as a scenario to stabilize the Higgs potential and to be consistent with electroweak naturalness $[28,29]$ is tested by the search for $\tilde{t}$ below the $\mathrm{TeV}$ scale.

In this Letter, we present a search for direct $\tilde{t}^{*}$ production assuming $\tilde{t} \rightarrow t \tilde{\chi}_{1}^{0} \rightarrow b W \tilde{\chi}_{1}^{0}$, where $\tilde{t}_{1}$ is the lightest $\tilde{t}$ eigenstate and $\tilde{\chi}_{1}^{0}$ represents the lightest supersymmetric particle (LSP) in $R$-parity conserving models [30-34]. We target events where both $W$ bosons decay hadronically, yielding a final state with six high transverse momentum $\left(p_{T}\right)$ jets from the all-hadronic $t \bar{t}$ final state and large missing transverse momentum $\left(E_{T}^{\text {miss }}\right)$ from the LSPs. The kinematics of both top quarks can therefore be fully specified by the visible decay products. Additionally, SM backgrounds from all-hadronic $t \bar{t}$ are suppressed as there is no intrinsic $E_{T}^{\text {miss }}$ except from semileptonic $c$ - and $b$-quark decays. The dominant background consists of $t \bar{t}$ events that contain a $W \rightarrow \ell \nu$ decay where the lepton $(\ell)$, often of $\tau$

*Full author list given at the end of the article.

Published by the American Physical Society under the terms of the Creative Commons Attribution 3.0 License. Further distribution of this work must maintain attribution to the author(s) and the published article's title, journal citation, and DOI. flavor, is either lost or misidentified as a jet. These events also have large $E_{T}^{\text {miss }}$ from the neutrino $(\nu)$.

The data were acquired during 2011 in LHC $p p$ collisions at a center-of-mass energy of $7 \mathrm{TeV}$ with the ATLAS detector [35], which consists of tracking detectors surrounded by a $2 \mathrm{~T}$ superconducting solenoid, calorimeters, and a muon spectrometer in a toroidal magnetic field. The high-granularity calorimeter system, with acceptance covering $|\eta|<4.9$ [36], is composed of liquid argon with lead, copper, or tungsten absorbers and scintillator tiles with steel absorbers. This data set, composed of events with a high- $p_{T}$ jet and large $E_{T}^{\text {miss }}$ as selected by the trigger system, corresponds to an integrated luminosity of $4.7 \mathrm{fb}^{-1}$ with a relative uncertainty of 3.9\% [37-39].

Jets are constructed from three-dimensional clusters of calorimeter cells using the anti- $k_{t}$ algorithm with a distance parameter of $0.4[39,40]$. Jet energies are corrected [41] for losses in material in front of the active calorimeter layers, detector inhomogeneities, the noncompensating nature of the calorimeter, and the impact of multiple overlapping $p p$ interactions. These corrections are derived from test beam, cosmic-ray, and $p p$ collision data, and from a detailed GEANT4 [42] detector simulation [43]. Jets containing a $b$-hadron are identified with an algorithm exploiting both the impact parameter and secondary vertex information $[44,45]$. A factor correcting for the slight differences in the $b$-tagging efficiency between data and the GEANT4 simulation is applied to each jet in the simulation. The $b$-jets are restricted to the fiducial region of the tracker, $|\eta|<2.5$. Non- $t \bar{t}$ backgrounds are minimized by requiring either $\geq 1 \quad b$-jets with a selection corresponding to a $60 \%$ efficiency with a low $<0.2 \%$ misidentification rate (tight), or $\geq 2 b$-jets each with $75 \%$ efficiency but a higher $\approx 1.7 \%$ misidentification rate per $b$-jet (loose).

The $E_{T}^{\text {miss }}$ is the magnitude of $\mathbf{p}_{T}^{\text {miss }}$, the negative vector sum of the $p_{T}$ of the clusters of calorimeter cells, calibrated according to their associated reconstructed object (e.g., jets and electrons), and of the $p_{T}$ of muons above $10 \mathrm{GeV}$ 
within $|\eta|<2$.4. Events containing $E_{T}^{\text {miss }}$ induced by jets associated with calorimeter noise or noncollision backgrounds [46], or by cosmic-ray muons [47,48], are removed from consideration. Large $\mathbf{p}_{T}^{\text {miss }}$ collinear with a high- $p_{T}$ jet could indicate a significant fluctuation in the reconstructed jet energy or the presence of a semileptonic $c$ - or $b$-quark decay. Therefore, the difference in azimuthal angle $(\Delta \phi)$ between the $\mathbf{p}_{t}^{\text {miss }}$ and any of the three highest- $p_{T}$ jets in the event, $\Delta \phi\left(\mathbf{p}_{T}^{\text {miss }}\right.$, jet $)$, is required to be $>\pi / 5$ radians. Fluctuations in the $E_{T}^{\text {miss }}$ are also suppressed by requiring that the $\Delta \phi$ between the above computed $\mathbf{p}_{T}^{\text {miss }}$ and one calculated with the tracking system, using tracks having $p_{T}>0.5 \mathrm{GeV}$, is $<\pi / 3$ radians.

Events are required to have at least one jet with $p_{T}>$ $130 \mathrm{GeV}$ in $|\eta|<2.8$ and $E_{T}^{\text {miss }}>150 \mathrm{GeV}$ to ensure full efficiency of the trigger. At least five other jets having $p_{T}>30 \mathrm{GeV}$ and $|\eta|<2.8$ must be present. In addition to the jet and $E_{T}^{\text {miss }}$ requirements, events containing "loose" electrons $[49,50]$ with $p_{T}>20 \mathrm{GeV}$ and $|\eta|<$ 2.47 that do not overlap with any jet within $\Delta R<0.4$, where $\Delta R=\sqrt{(\Delta \eta)^{2}+(\Delta \phi)^{2}}$, are rejected. Similarly, events with muons [47-51] having $p_{T}>10 \mathrm{GeV}$ and $|\eta|<2.4$ that are separated by $\Delta R>0.4$ from the nearest jet are rejected. A jet with 1-4 tracks and $\Delta \phi\left(\mathbf{p}_{T}^{\text {miss }}\right.$, jet $)<$ $\pi / 5$ indicates a likely $W \rightarrow \tau \nu$ decay. Events with such $\tau$-like jets that have transverse mass $m_{T}=\sqrt{2 p_{T} E_{T}^{\text {miss }}(1-\cos \Delta \phi)}<100 \mathrm{GeV}$ are rejected.

The presence of high- $p_{T}$ top quarks that decay through $t \rightarrow b W \rightarrow b j j$ in the $\tilde{t}_{1} \tilde{t}_{1}^{*}$ final state is exploited to further reduce SM backgrounds by only considering events with reconstructed three-jet invariant masses consistent with the top-quark mass $\left(m_{t}\right)$. A clustering technique resolves the combinatorics associated with high-multiplicity jet events. The three closest jets in the $\eta-\phi$ plane are combined to form one triplet; a second triplet is formed from the remaining jets by repeating the procedure. The resulting three-jet mass $\left(m_{j j j}\right)$ spectrum is shown in Fig. 1 for the control region constructed from $\ell+$ jets events (defined below). There is a clear peak associated with the hadronically decaying top quarks above a small non- $t \bar{t}$ background. A requirement of $80<m_{j j j}<270 \mathrm{GeV}$ is placed on each reconstructed triplet in the event. The kinematics of the $t \rightarrow b W \rightarrow b \ell \nu$ decay is also exploited to further reduce the dominant $\ell+$ jets $t \bar{t}$ background, as the $m_{T}$ distribution of the $\mathbf{p}_{T}^{\text {miss }}$ and $b$-jet $\left(m_{T}^{\text {jet }}\right.$ ) has an end point at $m_{t}$. When there are $\geq 2$ loose $b$-jets, the $m_{T}^{\text {jet }}$ for the $b$-jet closest to the $\mathbf{p}_{T}^{\text {miss }}$ is required to be $>175 \mathrm{GeV}$. The largest $m_{T}^{\text {jet }}$, calculated for each of the four highest- $p_{T}$ jets, is required to be $>175 \mathrm{GeV}$ in the case of only one tight $b$-jet.

Two signal regions (SR) are defined including the above kinematic and mass requirements. The first, which requires $E_{T}^{\text {miss }}>150 \mathrm{GeV}$ (SRA), is optimized for low $m_{\tilde{t}_{1}}$, while the second, requiring $E_{T}^{\text {miss }}>260 \mathrm{GeV}$ (SRB), is used for

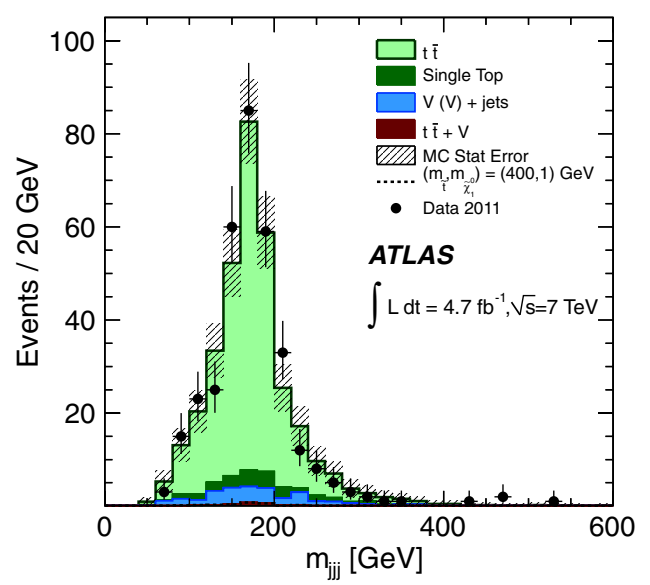

FIG. 1 (color online). Three-jet invariant mass distribution of the hadronic top-quark candidate in the control region constructed from $\ell+$ jets events where the signal expectation is minimal. Data are indicated by points; shaded histograms represent contributions from several SM sources (with $t \bar{t}$ scaled by 0.66 The hatched error bars indicate the total statistical uncertainty on the expected background. The distribution for the $m_{\tilde{t}_{1}}=400 \mathrm{GeV}, m_{\tilde{\chi}_{1}^{0}}=1 \mathrm{GeV}$ signal expectation in this control region is overlaid.

higher $m_{\tilde{t}_{1}}$. Using these signal regions, the search is most sensitive to $\tilde{t}_{1} \tilde{t}_{1}^{*}$ production with $350 \lesssim m_{\tilde{t}_{1}} \lesssim 500 \mathrm{GeV}$ and $m_{\tilde{\chi}_{1}^{0}} \ll m_{\tilde{t}_{1}}$. Signal events are simulated using HERWIG++ [52] with the MRST2007LO* [53] parton distribution functions (PDF) generated with the $\tilde{t}_{1}$ and $\tilde{\chi}_{1}^{0}$ masses at fixed values in a grid with $50 \mathrm{GeV}$ spacing. The mixing between $\tilde{t}_{L}$ and $\tilde{t}_{R}$ is chosen such that the lightest scalar top is mostly the partner of the right-handed top quark. The branching fraction of $\tilde{t} \rightarrow t \tilde{\chi}_{1}^{0}$ is set to $100 \%$ and the top-quark mass is set to $172.5 \mathrm{GeV}$. Signal cross sections are calculated to next-to-leading order in the strong coupling constant, including the resummation of soft gluon emission at next-to-leading-logarithmic accuracy (NLO + NLL) [10-12]. The nominal production cross section and associated uncertainty are taken from an envelope of cross section predictions using different PDF sets and factorization and renormalization scales, as described in Ref. [54]. The $\tilde{t}_{1} \tilde{t}_{1}^{*}$ cross section for $m_{\tilde{t}_{1}}=$ $400 \mathrm{GeV}$ is $\sigma_{\tilde{t}_{1} \tilde{T}_{1}^{*}}=0.21 \pm 0.03 \mathrm{pb}$.

In the signal region, the dominant source of SM background is $t \bar{t} \rightarrow \tau+$ jets events where the $\tau$ lepton is reconstructed as a jet. Additional, smaller, backgrounds include other $t \bar{t} \rightarrow \ell+$ jets final states, $t \bar{t}+V$ where $V$ represents a $W$ or $Z$ boson, single top-quark production, $V+$ jets, and $V V+$ jets. The $t \bar{t}$ events are produced with ALPGEN [55] using the CTEQ6L1 PDF [56] and interfaced to HERWIG [57,58] for particle production and JIMMY [59] for the underlying event model. TAUOLA was used to model the decay of $\tau$ leptons [60]. Additional $t \bar{t}$ samples generated with MC@NLO [61,62] and ACERMC [63], interfaced to HERWIG and JIMMY, are used to estimate event generator 
systematic uncertainties. Samples of $t \bar{t}+V$ are produced with MADGRAPH [64] interfaced to PYTHIA $[58,65,66]$. Single top events are generated with MC@NLO $[67,68]$ and ACERMC. The associated production of $W$ and $Z$ bosons and light and heavy-flavor jets is simulated using ALPGEN; diboson production is simulated with SHERPA [69].

All samples are passed through the GEANT4 simulation of the ATLAS detector, and are reconstructed in the same manner as the data. The simulation includes the effect of multiple $p p$ interactions and is weighted to reproduce the observed distribution of the number of interactions per bunch crossing. SM event samples are normalized to the results of higher-order calculations using the cross sections cited in Ref. [70] except for the $\ell+$ jets $t \bar{t}$ background. This sample is normalized by a factor that scales the $t \bar{t}$ expectation to agree with the observed data in a control region (CR) kinematically close to the signal region but with little expected signal. The CR is constructed from events containing one muon or one "tight" electron [49] with $p_{T}>$ $30 \mathrm{GeV}$ consistent with originating from a $W$-boson decay $\left(40<m_{T}^{\ell}<120 \mathrm{GeV}\right)$ and $\geq 5$ jets, where $m_{T}^{\ell}$ is the transverse mass of the electron or muon and $\mathbf{p}_{T}^{\text {miss }}$. The lepton must be isolated such that the scalar $p_{T}$ sum of tracks in a cone of $\Delta R<0.2$ around the lepton, excluding the track of the lepton, is $<1.8 \mathrm{GeV}$ for the muon or is $<10 \%$ of the electron $p_{T}$, respectively. The jet, $b$-jet, and $E_{T}^{\text {miss }}$ requirements remain the same as the standard signal selection; however, some topological constraints are relaxed $\left[\Delta \phi\left(\mathbf{p}_{T}^{\text {miss }}\right.\right.$, jet $)>\pi / 10$ radians and $\left.m_{j j j}<600 \mathrm{GeV}\right]$ and others removed $\left(m_{T}^{\text {jet }}\right)$ to gain statistics. The $t \bar{t}$ purity in the control region is $>80 \%$; the expected signal contamination is $<3 \%$. The lepton is treated as a jet of the same energy and momentum, mimicking the effect of the $\tau$ lepton. Effects of the additional $E_{T}^{\text {miss }}$ from the $\tau$ neutrino are smaller than the statistical uncertainties. The normalization is scaled by $0.66 \pm 0.05$ to bring the $\geq 6$ jet $\ell+$ jets ALPGEN $t \bar{t}$ events into agreement with the data after recalculating all quantities except $E_{T}^{\text {miss; }}$; the uncertainty quoted here is statistical only. This scale factor is used in Figs. 1-3. The normalization is validated with an orthogonal $t \bar{t}$-dominated sample created from SRA by selecting events with $\tau$-like jets; the requirement on $m_{T}^{\text {jet }}$ is removed to increase the sample size. The $m_{T}$ of $\tau$-like jets is shown in Fig. 2, where the $t \bar{t}$ sample has been normalized as described above. Expectations from the simulation agree with the data within uncertainties. Contributions to the signal region from QCD multijet and all-hadronic $t \bar{t}$ production are estimated with a data-driven technique [71]. Jets are smeared in a low- $E_{T}^{\text {miss }}$ data sample using response functions derived from control regions dominated by multijet events. The expected number of such events is $0.2 \pm 0.2$ in SRA after the full event selection.

The $E_{T}^{\text {miss }}$ distribution in SRA is shown in Fig. 3 for data, for the SM backgrounds, and for expectations of $\tilde{t}_{1} \tilde{t}_{1}^{*}$ production with $m_{\tilde{t}_{1}}=400$ and $m_{\tilde{\chi}_{1}^{0}}=1 \mathrm{GeV}$. Numbers

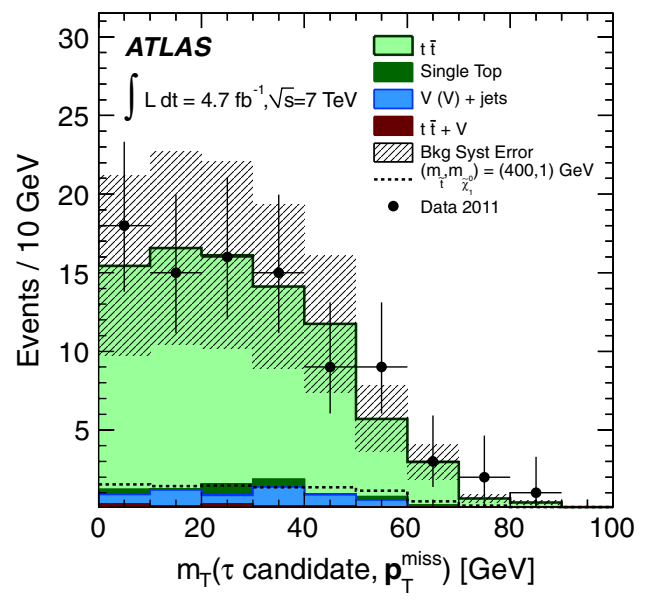

FIG. 2 (color online). The $m_{T}$ distribution for $\tau$-like jets with the selection described in the text. Data are indicated by points; shaded histograms represent contributions from several SM sources (with $t \bar{t}$ scaled by 0.66 ). The hatched error bars indicate the systematic uncertainty on the total expected background. The expected signal distribution for $m_{\tilde{t}_{1}}=400 \mathrm{GeV}, m_{\tilde{\chi}_{1}^{0}}=1 \mathrm{GeV}$ is overlaid.

of events and combined statistical and systematic uncertainties, for both SRA and SRB, are tabulated in Table I. Uncertainties in the event generators, including the impact of initial- and final-state radiation, are the dominant source of systematic uncertainty of $28 \%(23 \%)$ for the background in SRA (SRB). Other major sources of uncertainty include $22 \%(32 \%)$ for the jet energy calibration, $6.5 \%(6.8 \%)$ for jet energy resolution, $5.9 \%$ (6.2\%) for $b$-jet identification, and $1.4 \%(1.5 \%)$ for $E_{T}^{\text {miss }}$ in SRA (SRB).

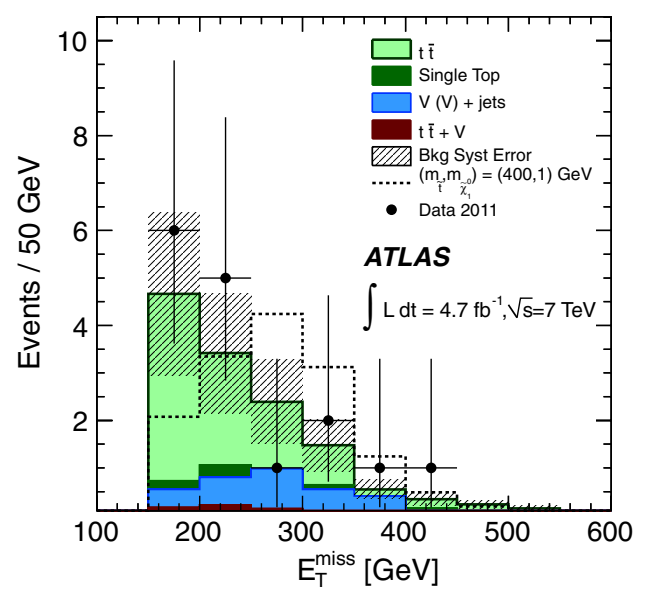

FIG. 3 (color online). The $E_{\mathrm{T}}^{\text {miss }}$ distribution in data compared to the SM expectation for signal region $\mathrm{A}$. The hatched error bars indicate the systematic uncertainty on the total expected background. The expected signal distribution for $m_{\tilde{t}_{1}}=400 \mathrm{GeV}$, $m_{\tilde{\chi}_{1}^{0}}=1 \mathrm{GeV}$ is overlaid. The SM background distributions do not include the $0.2 \pm 0.2$ events of all-hadronic $t \bar{t}$ and QCD multijets estimated from data. 
TABLE I. The numbers of expected events for the SM backgrounds and an example SUSY signal point, and the observed number of events in data. The $95 \%$ C.L. upper limit on the observed (expected) visible cross section, as defined in the text, is appended below.

\begin{tabular}{lcc}
\hline \hline & SRA & SRB \\
& $E_{\mathrm{T}}^{\text {miss }}>150 \mathrm{GeV}$ & $>260 \mathrm{GeV}$ \\
\hline$t \bar{t}$ & $9.2 \pm 2.7$ & $2.3 \pm 0.6$ \\
$t \bar{t}+W / Z$ & $0.8 \pm 0.2$ & $0.4 \pm 0.1$ \\
Single top & $0.7 \pm 0.4$ & $0.2_{-0.2}^{+0.3}$ \\
$Z+$ jets & $1.3_{-1.0}^{+1.1}$ & $0.9_{-0.7}^{+0.8}$ \\
$W+$ jets & $1.2_{-1.0}^{+1.4}$ & $0.5 \pm 0.4$ \\
Diboson & $0.1_{-0.1}^{+0.2}$ & $0.1_{-0.1}^{+0.2}$ \\
Multijets & $0.2^{+0.2}$ & $0.02 \pm 0.02$ \\
Total SM & $13.5_{-3.6}^{+3.7}$ & $4.4_{-1.3}^{+1.7}$ \\
SUSY $\left(m_{\tilde{t}_{1}}, m_{\tilde{\chi}_{1}}\right)=(400,1) \mathrm{GeV}$ & $14.8^{ \pm} 4.0$ & $8.9 \pm 3.1$ \\
Data $($ observed) & $16^{2}$ & 4 \\
Visible cross section limit $[\mathrm{fb}]$ & $2.9(2.5)$ & $1.3(1.3)$ \\
\hline \hline
\end{tabular}

The number of observed events in the data is well matched by the SM background. These results are interpreted as exclusion limits for $m_{\tilde{t}_{1}}$ and $m_{\tilde{\chi}_{1}^{0}}$ using a $\mathrm{CL}_{s}$ likelihood ratio combining Poisson probabilities for signal and background [72]. Systematic uncertainties are treated

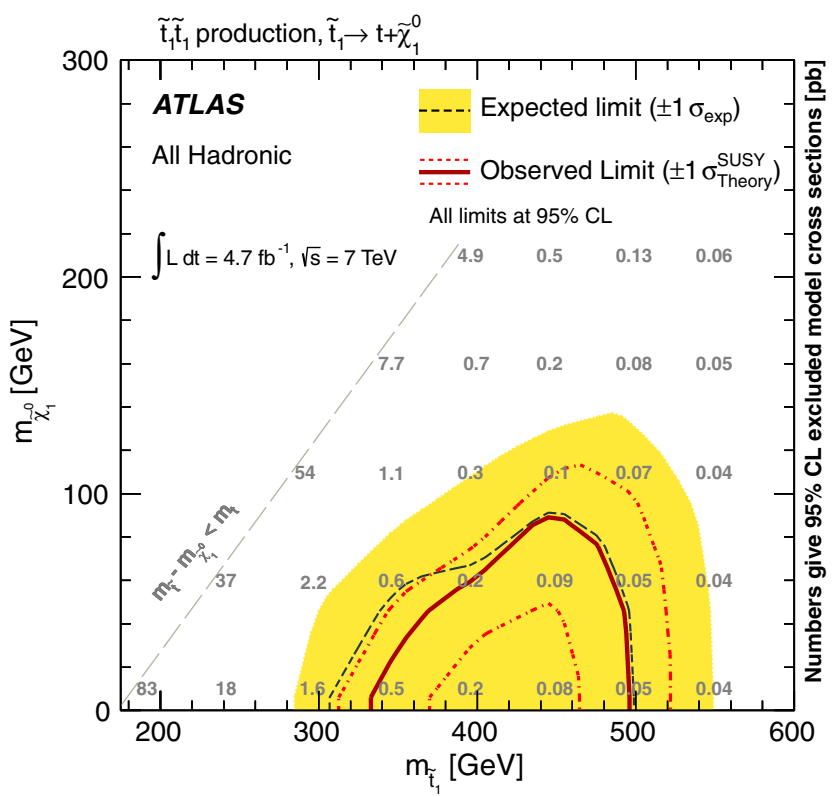

FIG. 4 (color online). Expected and observed 95\% C.L. exclusion limits in the plane of $m_{\tilde{\chi}_{1}^{0}}$ vs $m_{\tilde{t}_{1}}$, assuming $100 \%$ branching fraction for $\tilde{t}_{1} \rightarrow t \tilde{\chi}_{1}^{0}$. The dashed line shows the expected limit at $95 \%$ C.L. with the shaded region indicating the $\pm 1 \sigma$ exclusions due to experimental uncertainties. Observed limits are indicated by the solid contour (nominal) and the dotted contours (obtained by varying the SUSY cross section by the theoretical uncertainties). The inner dotted contour indicates the excluded region. The dashed diagonal line represents the kinematic limit for the $t \tilde{\chi}_{1}^{0}$ final state. The numbers overlaid on the plot represent the $95 \%$ C.L. excluded visible cross sections in pb. as nuisance parameters assuming Gaussian distributions. Uncertainties associated with jets, $b$-jets, $E_{T}^{\text {miss }}$, and luminosity are fully correlated between signal and background; the others are assumed to be uncorrelated. The expected limits for the signal regions are evaluated for each $\left(m_{\tilde{t}_{1}}\right.$, $\left.m_{\tilde{\chi}_{1}^{0}}\right)$ point; the SR with the better expected sensitivity is used for that point. The expected and observed 95\% C.L. exclusion limits, interpolating across points, are displayed in Fig. 4. The $-1 \sigma$ observed limit contour that accounts for theoretical uncertainties on the SUSY cross sections is maximum at $\left(m_{\tilde{t}_{1}}, m_{\tilde{\chi}_{1}^{0}}\right)=(445,50) \mathrm{GeV}$. Top squark masses between 370 and $465 \mathrm{GeV}$ are excluded for $m_{\tilde{\chi}_{1}^{0}} \sim$ $0 \mathrm{GeV}$. These values are derived from the $-1 \sigma$ observed limit contour to account for theoretical uncertainties on the SUSY cross sections. The 95\% C.L. upper limit on the number of events beyond the SM in each signal region, divided by the integrated luminosity, yields limits on the observed (expected) visible cross sections of 2.9 (2.5) fb in SRA and 1.3 (1.3) fb in SRB.

In conclusion, we have presented a search for the direct production of $\tilde{t}_{1} \tilde{t}_{1}^{*}$ in the $t \tilde{\chi}_{1}^{0} \bar{\tau} \tilde{\chi}_{1}^{0}$ decay channel, assuming $100 \%$ branching fraction for $\tilde{t}_{1} \rightarrow t \tilde{\chi}_{1}^{0}$. A total of 16 (4) events are observed compared to a predicted standard model background of $13.5_{-3.6}^{+3.7}\left(4.4_{-1.3}^{+1.7}\right)$ events in two signal regions based on $\int \mathcal{L} d t=4.7 \mathrm{fb}^{-1}$ of $p p$ collision data taken at $\sqrt{s}=7 \mathrm{TeV}$ with the ATLAS detector at the LHC. No evidence for $\tilde{t}_{1} \tilde{t}_{1}^{*}$ is observed in data and $95 \%$ C.L. limits are set on $\tilde{t}_{1} \tilde{t}_{1}^{*}$ production as a function of $m_{\tilde{t}_{1}}$ and $m_{\tilde{\chi}_{1}^{0}}$.

We thank CERN for the very successful operation of the LHC, as well as the support staff from our institutions without whom ATLAS could not be operated efficiently. We acknowledge the support of ANPCyT, Argentina; YerPhI, Armenia; ARC, Australia; BMWF, Austria; ANAS, Azerbaijan; SSTC, Belarus; CNPq and FAPESP, Brazil; NSERC, NRC, and CFI, Canada; CERN; CONICYT, Chile; CAS, MOST, and NSFC, China; COLCIENCIAS, Colombia; MSMT CR, MPO CR, and VSC CR, Czech Republic; DNRF, DNSRC, and Lundbeck Foundation, Denmark; EPLANET and ERC, European Union; IN2P3-CNRS and CEA-DSM/IRFU, France; GNAS, Georgia; BMBF, DFG, HGF, MPG, and AvH Foundation, Germany; GSRT, Greece; ISF, MINERVA, GIF, DIP, and Benoziyo Center, Israel; INFN, Italy; MEXT and JSPS, Japan; CNRST, Morocco; FOM and NWO, Netherlands; RCN, Norway; MNiSW, Poland; GRICES and FCT, Portugal; MERYS (MECTS), Romania; MES of Russia and ROSATOM, Russian Federation; JINR; MSTD, Serbia; MSSR, Slovakia; ARRS and MVZT, Slovenia; DST/NRF, South Africa; MICINN, Spain; SRC and Wallenberg Foundation, Sweden; SER, SNSF, and Cantons of Bern and Geneva, Switzerland; NSC, Taiwan; TAEK, Turkey; STFC, the Royal Society, and Leverhulme Trust, United Kingdom; DOE and NSF, United States of America. The crucial com- 
puting support from all WLCG partners is acknowledged gratefully, in particular, from CERN and the ATLAS Tier-1 facilities at TRIUMF (Canada), NDGF (Denmark, Norway, Sweden), CC-IN2P3 (France), KIT/GridKA (Germany), INFN-CNAF (Italy), NL-T1 (Netherlands), PIC (Spain), ASGC (Taiwan), RAL (UK), and BNL (USA) and in the Tier-2 facilities worldwide.

[1] H. Miyazawa, Prog. Theor. Phys. 36, 1266 (1966).

[2] P. Ramond, Phys. Rev. D 3, 2415 (1971).

[3] Y. A. Gol'fand and E. P. Likhtman, Pis'ma Zh. Eksp. Teor. Fiz. 13, 452 (1971) [JETP Lett. 13, 323 (1971)].

[4] A. Neveu and J. H. Schwarz, Nucl. Phys. B31, 86 (1971).

[5] A. Neveu and J. H. Schwarz, Phys. Rev. D 4, 1109 (1971).

[6] J. Gervais and B. Sakita, Nucl. Phys. B34, 632 (1971).

[7] D. V. Volkov and V.P. Akulov, Phys. Lett. 46B, 109 (1973).

[8] J. Wess and B. Zumino, Phys. Lett. 49B, 52 (1974).

[9] J. Wess and B. Zumino, Nucl. Phys. B70, 39 (1974).

[10] W. Beenakker, M. Krämer, T. Plehn, M. Spira, and P. M. Zerwas, Nucl. Phys. B515, 3 (1998).

[11] W. Beenakker, S. Brensing, M. Krämer, A. Kulesza, E. Laenen, and I. Niessen, J. High Energy Phys. 08 (2010) 098.

[12] W. Beenakker, S. Brensing, M. Krämer, A. Kulesza, and E. Laenen, Int. J. Mod. Phys. A 26, 2637 (2011).

[13] P. Achard et al. (L3 Collaboration), Phys. Lett. B 580, 37 (2004).

[14] J. Abdallah et al. (DELPHI Collaboration), Eur. Phys. J. C 31, 421 (2003).

[15] G. Abbiendi et al. (OPAL Collaboration), Phys. Lett. B 545, 272 (2002).

[16] A. Heister et al. (ALEPH Collaboration), Phys. Lett. B 537, 5 (2002).

[17] V. M. Abazov et al. (D0 Collaboration), Phys. Lett. B 710, 578 (2012).

[18] V. M. Abazov et al. (D0 Collaboration), Phys. Lett. B 696, 321 (2011).

[19] T. Aaltonen et al. (CDF Collaboration), Phys. Rev. Lett. 104, 251801 (2010).

[20] V. Abazov et al. (D0 Collaboration), Phys. Lett. B 674, 4 (2009).

[21] V. Abazov et al. (D0 Collaboration), Phys. Lett. B 675, 289 (2009).

[22] T. Aaltonen et al. (CDF Collaboration), Phys. Rev. Lett. 101, 071802 (2008).

[23] V. Abazov et al. (D0 Collaboration), Phys. Lett. B 665, 1 (2008).

[24] T. Aaltonen et al. (CDF Collaboration), Phys. Rev. D 76, 072010 (2007).

[25] D. Acosta et al. (CDF Collaboration), Phys. Rev. Lett. 92, 051803 (2004).

[26] ATLAS Collaboration, Phys. Lett. B 715, 44 (2012).

[27] ATLAS Collaboration, Report No. ATLAS-CONF-2012059, 2011, http://cdsweb.cern.ch/record/1453787.

[28] R. Barbieri and G. Giudice, Nucl. Phys. B306, 63 (1988).

[29] B. de Carlos and J. Casas, Phys. Lett. B 309, 320 (1993).

[30] P. Fayet, Phys. Lett. 64B, 159 (1976).

[31] P. Fayet, Phys. Lett. 69B, 489 (1977).
[32] G. R. Farrar and P. Fayet, Phys. Lett. 76B, 575 (1978).

[33] P. Fayet, Phys. Lett. 84B, 416 (1979).

[34] S. Dimopoulos and H. Georgi, Nucl. Phys. B193, 150 (1981).

[35] ATLAS Collaboration, JINST 3, S08003 (2008).

[36] ATLAS uses a right-handed coordinate system with its origin at the nominal interaction point in the center of the detector and the $z$ axis along the beam pipe. Cylindrical coordinates $(r, \phi)$ are used in the transverse plane, being the azimuthal angle around the beam pipe. The pseudorapidity is defined in terms of the polar angle $\theta$ as $\eta=-\ln \tan (\theta / 2)$.

[37] ATLAS Collaboration, Eur. Phys. J. C 71, 1630 (2011).

[38] ATLAS Collaboration, Report No. ATLAS-CONF-2011116, 2011, http://cdsweb.cern.ch/record/1376384.

[39] M. Cacciari, G. P. Salam, and G. Soyez, J. High Energy Phys. 04 (2008) 063.

[40] M. Cacciari and G. P. Salam, Phys. Lett. B 641, 57 (2006).

[41] ATLAS Collaboration, arXiv:1112.6426 [Eur. Phys. J. C (to be published)].

[42] S. Agostinelli et al. (GEANT4 Collaboration), Nucl. Instrum. Methods Phys. Res., Sect. A 506, 250 (2003).

[43] ATLAS Collaboration, Eur. Phys. J. C 70, 823 (2010).

[44] ATLAS Collaboration, Report No. ATLAS-CONF-2012043, 2012, MV1 algorithm, http://cdsweb.cern.ch/record/ 1435197.

[45] ATLAS Collaboration, Report No. ATLAS-CONF-2012040 (2012), MV1 algorithm, http://cdsweb.cern.ch/record/ 1435194.

[46] ATLAS Collaboration, Report No. ATLAS-CONF-2012020, 2012, http://cdsweb.cern.ch/record/1430034.

[47] ATLAS Collaboration, Report No. ATLAS-CONF-2011063, 2011, http://cdsweb.cern.ch/record/1345743.

[48] ATLAS Collaboration, Eur. Phys. J. C 71, 1593 (2011).

[49] ATLAS Collaboration, Eur. Phys. J. C 72, 1909 (2012).

[50] ATLAS Collaboration, Report No. ATLAS-COMPHYS-2011-1669, 2011, http://atlas.web.cern.ch/Atlas/ GROUPS/PHYSICS/EGAMMA/PublicPlots/20111412/ EfficiencyVsET/ATL-COM-PHYS-2011-1669/index. html.

[51] ATLAS Collaboration, Report No. ATLAS-CONF-2011021, 2011, http://cdsweb.cern.ch/record/1336750.

[52] M. Bähr et al., Eur. Phys. J. C 58, 639 (2008), HERWIG++ V2.4.2.

[53] A. Sherstnev and R. S. Thorne, Eur. Phys. J. C 55, 553 (2008).

[54] M. Krämer, A. Kulesza, R. van der Leeuw, M. Mangano, S. Padhi, T. Plehn, and X. Portell, arXiv:1206.2892.

[55] M. L. Mangano, M. Moretti, F. Piccinini, R. Pittau, and A. D. Polosa, J. High Energy Phys. 07 (2003) 001, ALPGEN V2.13.

[56] J. Pumplin, D. Stump, J. Huston, H. Lai, P. Nadolsky, and W. Tung, J. High Energy Phys. 07 (2002) 012.

[57] G. Corcella, I. Knowles, G. Marchesini, S. Moretti, K. Odagiri, P. Richardson, M. Seymour, and B. Webber, J. High Energy Phys. 01 (2001) 010.

[58] ATLAS Collaboration, Report No. ATLAS-PUB-2011008 (2011), http://cdsweb.cern.ch/record/1345343.

[59] J. Butterworth, J. R. Forshaw, and M. Seymour, Z. Phys. C 72, 637 (1996), JIMMY V4.31. 
[60] S. Jadach, Z. Was, R. Decker, and J.H. Kuhn, Comput. Phys. Commun. 76, 361 (1993).

[61] S. Frixione and B. R. Webber, J. High Energy Phys. 06 (2002) 029.

[62] S. Frixione, P. Nason, and B. R. Webber, J. High Energy Phys. 08 (2003) 007, MC@NLO V3.41.

[63] B.P. Kersevan and E. Richter-Was, ACERMC V3.7, arXiv: hep-ph/0405247.

[64] J. Alwall, P. Demin, S. de Visscher, R. Frederix, M. Herquet, F. Maltoni, T. Plehn, D. Rainwater, and T. Stelzer, J. High Energy Phys. 09 (2007) 028.

[65] T. Sjöstrand, S. Mrenna, and P.Z. Skands, J. High Energy Phys. 05 (2006) 026.
[66] ATLAS Collaboration, Report No. ATLAS-PUB-2011014, 2011, http://cdsweb.cern.ch/record/1400677.

[67] S. Frixione, E. Laenen, P. Motylinski, and B. R. Webber, J. High Energy Phys. 03 (2006) 092.

[68] S. Frixione, E. Laenen, P. Motylinski, B. R. Webber, and C. D. White, J. High Energy Phys. 07 (2008) 029.

[69] T. Gleisberg, S. Hoeche, F. Krauss, M. Schonherr, S. Schumann, F. Siegert, and J. Winter, J. High Energy Phys. 02 (2009) 007, SHERPA V1.2.3.

[70] ATLAS Collaboration, arXiv:1208.0949.

[71] ATLAS Collaboration, Phys. Lett. B 710, 67 (2012).

[72] A. L. Read, J. Phys. G 28, 2693 (2002).

G. Aad ${ }^{48}$ T. Abajyan, ${ }^{21}$ B. Abbott, ${ }^{111}$ J. Abdallah, ${ }^{12}$ S. Abdel Khalek, ${ }^{115}$ A. A. Abdelalim, ${ }^{49}$ O. Abdinov, ${ }^{11}$ R. Aben, ${ }^{105}$ B. Abi, ${ }^{112}$ M. Abolins, ${ }^{88}$ O. S. AbouZeid, ${ }^{158}$ H. Abramowicz, ${ }^{153}$ H. Abreu, ${ }^{136}$ B. S. Acharya, ${ }^{164 a, 164 b}$ L. Adamczyk, ${ }^{38}$ D. L. Adams, ${ }^{25}$ T. N. Addy ${ }^{56}$ J. Adelman, ${ }^{176}$ S. Adomeit,${ }^{98}$ P. Adragna, ${ }^{75}$ T. Adye, ${ }^{129}$ S. Aefsky, ${ }^{23}$ J. A. Aguilar-Saavedra, ${ }^{124 b, b}$ M. Agustoni, ${ }^{17}$ M. Aharrouche, ${ }^{81}$ S. P. Ahlen, ${ }^{22}$ F. Ahles, ${ }^{48}$ A. Ahmad, ${ }^{148}$ M. Ahsan, ${ }^{41}$ G. Aielli, ${ }^{133 a, 133 b}$ T. Akdogan, ${ }^{19 a}$ T. P. A. Åkesson, ${ }^{79}$ G. Akimoto, ${ }^{155}$ A. V. Akimov, ${ }^{94}$ M. S. Alam, ${ }^{2}$ M. A. Alam, ${ }^{76}$ J. Albert, ${ }^{169}$ S. Albrand, ${ }^{55}$ M. Aleksa, ${ }^{30}$ I. N. Aleksandrov, ${ }^{64}$ F. Alessandria, ${ }^{89 a}$ C. Alexa, ${ }^{26 a}$ G. Alexander, ${ }^{153}$ G. Alexandre, ${ }^{49}$ T. Alexopoulos ${ }^{10}$ M. Alhroob, ${ }^{164 a, 164 \mathrm{c}}$ M. Aliev, ${ }^{16}$ G. Alimonti, ${ }^{89 a}$ J. Alison, ${ }^{120}$

B. M. M. Allbrooke, ${ }^{18}$ P. P. Allport,${ }^{73}$ S. E. Allwood-Spiers, ${ }^{53}$ J. Almond, ${ }^{82}$ A. Aloisio, ${ }^{102 a, 102 b}$ R. Alon, ${ }^{172}$ A. Alonso, ${ }^{79}$ F. Alonso, ${ }^{70}$ A. Altheimer,${ }^{35}$ B. Alvarez Gonzalez,${ }^{88}$ M. G. Alviggi, ${ }^{102 a, 102 b}$ K. Amako, ${ }^{65}$ C. Amelung, ${ }^{23}$ V. V. Ammosov, ${ }^{128, a}$ S. P. Amor Dos Santos,${ }^{124 a}$ A. Amorim, ${ }^{124 a, c}$ N. Amram, ${ }^{153}$ C. Anastopoulos,${ }^{30}$ L. S. Ancu, ${ }^{17}$ N. Andari, ${ }^{115}$ T. Andeen, ${ }^{35}$ C. F. Anders, ${ }^{58 b}$ G. Anders ${ }^{58 a}$ K. J. Anderson, ${ }^{31}$ A. Andreazza, ${ }^{89 a, 89 b}$ V. Andrei ${ }^{58 a}$ X. S. Anduaga,${ }^{70}$ P. Anger, ${ }^{44}$ A. Angerami,,${ }^{35}$ F. Anghinolfi, ${ }^{30}$ A. Anisenkov, ${ }^{107}$ N. Anjos, ${ }^{124 a}$ A. Annovi ${ }^{47}$ A. Antonaki,${ }^{9}$ M. Antonelli, ${ }^{47}$ A. Antonov, ${ }^{96}$ J. Antos, ${ }^{144 b}$ F. Anulli, ${ }^{132 a}$ M. Aoki, ${ }^{101}$ S. Aoun, ${ }^{83}$ L. Aperio Bella, ${ }^{5}$ R. Apolle, ${ }^{118, \mathrm{~d}}$ G. Arabidze, ${ }^{88}$ I. Aracena, ${ }^{143}$ Y. Arai,${ }^{65}$ A. T. H. Arce, ${ }^{45}$ S. Arfaoui, ${ }^{148}$ J-F. Arguin, ${ }^{15}$ E. Arik, ${ }^{19 a, a}$ M. Arik, ${ }^{19 a}$ A. J. Armbruster, ${ }^{87}$ O. Arnaez, ${ }^{81}$ V. Arnal,${ }^{80}$ C. Arnault, ${ }^{115}$ A. Artamonov, ${ }^{95}$ G. Artoni, ${ }^{132 a, 132 b}$ D. Arutinov, ${ }^{21}$ S. Asai, ${ }^{155}$ R. Asfandiyarov, ${ }^{173}$ S. Ask, ${ }^{28}$ B. Åsman, ${ }^{146 a, 146 b}$ L. Asquith, ${ }^{6}$ K. Assamagan, ${ }^{25}$ A. Astbury, ${ }^{169}$ M. Atkinson, ${ }^{165}$ B. Aubert, ${ }^{5}$ E. Auge, ${ }^{115}$ K. Augsten, ${ }^{127}$ M. Aurousseau, ${ }^{145 a}$ G. Avolio, ${ }^{163}$ R. Avramidou, ${ }^{10}$ D. Axen, ${ }^{168}$ G. Azuelos, ${ }^{93, e}$ Y. Azuma, ${ }^{155}$ M. A. Baak, ${ }^{30}$ G. Baccaglioni,${ }^{89 a}$ C. Bacci, ${ }^{134 a, 134 b}$ A. M. Bach, ${ }^{15}$ H. Bachacou, ${ }^{136}$ K. Bachas,${ }^{30}$ M. Backes,${ }^{49}$ M. Backhaus, ${ }^{21}$ E. Badescu, ${ }^{26 a}$ P. Bagnaia, ${ }^{132 a, 132 b}$ S. Bahinipati, ${ }^{3}$ Y. Bai, ${ }^{33 a}$ D. C. Bailey, ${ }^{158}$ T. Bain, ${ }^{158}$ J. T. Baines, ${ }^{129}$ O. K. Baker,${ }^{176}$ M. D. Baker, ${ }^{25}$ S. Baker, ${ }^{77}$ E. Banas, ${ }^{39}$ P. Banerjee, ${ }^{93}$ Sw. Banerjee, ${ }^{173}$ D. Banfi, ${ }^{30}$ A. Bangert,${ }^{150}$ V. Bansal,,${ }^{169}$ H. S. Bansil, ${ }^{18}$ L. Barak, ${ }^{172}$ S. P. Baranov,${ }^{94}$ A. Barbaro Galtieri, ${ }^{15}$ T. Barber,${ }^{48}$ E. L. Barberio,${ }^{86}$ D. Barberis, ${ }^{50 a, 50 b}$ M. Barbero, ${ }^{21}$ D. Y. Bardin, ${ }^{64}$ T. Barillari, ${ }^{99}$ M. Barisonzi, ${ }^{175}$ T. Barklow, ${ }^{143}$ N. Barlow, ${ }^{28}$ B. M. Barnett, ${ }^{129}$ R. M. Barnett, ${ }^{15}$ A. Baroncelli, ${ }^{134 a}$ G. Barone,${ }^{49}$ A. J. Barr, ${ }^{118}$ F. Barreiro, ${ }^{80}$ J. Barreiro Guimarães da Costa ${ }^{57}$ P. Barrillon, ${ }^{115}$ R. Bartoldus, ${ }^{143}$ A. E. Barton, ${ }^{71}$ V. Bartsch, ${ }^{149}$ A. Basye, ${ }^{165}$ R. L. Bates,${ }^{53}$ L. Batkova, ${ }^{144 a}$ J. R. Batley, ${ }^{28}$ A. Battaglia, ${ }^{17}$ M. Battistin, ${ }^{30}$ F. Bauer, ${ }^{136}$ H. S. Bawa, ${ }^{143, f}$ S. Beale,${ }^{98}$ T. Beau, ${ }^{78}$ P. H. Beauchemin, ${ }^{161}$ R. Beccherle ${ }^{50 a}$ P. Bechtle,${ }^{21}$ H. P. Beck, ${ }^{17}$ A. K. Becker, ${ }^{175}$ S. Becker, ${ }^{98}$ M. Beckingham, ${ }^{138}$ K. H. Becks, ${ }^{175}$ A. J. Beddall, ${ }^{19 \mathrm{c}}$ A. Beddall, ${ }^{19 \mathrm{c}}$ S. Bedikian, ${ }^{176}$ V. A. Bednyakov, ${ }^{64}$ C. P. Bee,${ }^{83}$ L. J. Beemster, ${ }^{105}$ M. Begel, ${ }^{25}$ S. Behar Harpaz, ${ }^{152}$ M. Beimforde, ${ }^{99}$ C. Belanger-Champagne, ${ }^{85}$ P. J. Bell,${ }^{49}$ W. H. Bell, ${ }^{49}$ G. Bella,,${ }^{153}$ L. Bellagamba, ${ }^{20 \mathrm{a}}$ F. Bellina, ${ }^{30} \mathrm{M}$. Bellomo, ${ }^{30}$ A. Belloni, ${ }^{57}$ O. Beloborodova, ${ }^{107, \mathrm{~g}} \mathrm{~K}$. Belotskiy, ${ }^{96} \mathrm{O}$. Beltramello, ${ }^{30}$ O. Benary, ${ }^{153}$ D. Benchekroun, ${ }^{135 a}$ K. Bendtz, ${ }^{146 a, 146 b}$ N. Benekos, ${ }^{165}$ Y. Benhammou, ${ }^{153}$ E. Benhar Noccioli ${ }^{49}$ J. A. Benitez Garcia, ${ }^{159 b}$ D. P. Benjamin, ${ }^{45}$ M. Benoit, ${ }^{115}$ J. R. Bensinger, ${ }^{23}$ K. Benslama, ${ }^{130}$ S. Bentvelsen, ${ }^{105}$ D. Berge ${ }^{30}$ E. Bergeaas Kuutmann, ${ }^{42}$ N. Berger,${ }^{5}$ F. Berghaus, ${ }^{169}$ E. Berglund, ${ }^{105}$ J. Beringer, ${ }^{15}$ P. Bernat,${ }^{77}$ R. Bernhard ${ }^{48}$ C. Bernius, ${ }^{25}$ T. Berry, ${ }^{76}$ C. Bertella, ${ }^{83}$ A. Bertin, ${ }^{20 a, 20 b}$ F. Bertolucci, ${ }^{122 a, 122 b}$ M. I. Besana, ${ }^{89 a, 89 b}$ G. J. Besjes, ${ }^{104}$ N. Besson, ${ }^{136}$ S. Bethke, ${ }^{99}$ W. Bhimji, ${ }^{46}$ R. M. Bianchi,${ }^{30}$ M. Bianco, ${ }^{72 a, 72 b}$ O. Biebel,${ }^{98}$ S. P. Bieniek, ${ }^{77}$ K. Bierwagen,${ }^{54}$ J. Biesiada ${ }^{15}$ M. Biglietti, ${ }^{134 a}$ H. Bilokon, ${ }^{47}$ M. Bindi, ${ }^{20 a, 20 b}$ S. Binet, ${ }^{115}$ A. Bingul, ${ }^{19 \mathrm{c}}$ C. Bini, ${ }^{132 a, 132 \mathrm{~b}}$ C. Biscarat, ${ }^{178}$ B. Bittner,${ }^{99}$ K. M. Black,${ }^{22}$ R. E. Blair, ${ }^{6}$ J.-B. Blanchard, ${ }^{136}$ G. Blanchot ${ }^{30}$ T. Blazek, ${ }^{144 a}$ I. Bloch,${ }^{42}$ C. Blocker,${ }^{23}$ J. Blocki, ${ }^{39}$ A. Blondel, ${ }^{49}$ W. Blum, ${ }^{81}$ U. Blumenschein, ${ }^{54}$ 
G. J. Bobbink, ${ }^{105}$ V. B. Bobrovnikov, ${ }^{107}$ S. S. Bocchetta, ${ }^{79}$ A. Bocci ${ }^{45}$ C. R. Boddy ${ }^{118}$ M. Boehler ${ }^{48}$ J. Boek, ${ }^{175}$ N. Boelaert, ${ }^{36}$ J. A. Bogaerts, ${ }^{30}$ A. Bogdanchikov, ${ }^{107}$ A. Bogouch, ${ }^{90, a}$ C. Bohm, ${ }^{146 a}$ J. Bohm, ${ }^{125}$ V. Boisvert, ${ }^{76}$ T. Bold, ${ }^{38}$ V. Boldea, ${ }^{26 a}$ N. M. Bolnet, ${ }^{136}$ M. Bomben, ${ }^{78}$ M. Bona, ${ }^{75}$ M. Boonekamp, ${ }^{136}$ S. Bordoni, ${ }^{78}$ C. Borer, ${ }^{17}$ A. Borisov, ${ }^{128}$ G. Borissov, ${ }^{71}$ I. Borjanovic, ${ }^{13 a}$ M. Borri, ${ }^{82}$ S. Borroni, ${ }^{87}$ V. Bortolotto, ${ }^{134 a, 134 b}$ K. Bos, ${ }^{105}$ D. Boscherini, ${ }^{20 \mathrm{a}} \mathrm{M}$. Bosman, ${ }^{12} \mathrm{H}$. Boterenbrood, ${ }^{105} \mathrm{~J}$. Bouchami, ${ }^{93} \mathrm{~J}$. Boudreau, ${ }^{123}$ E. V. Bouhova-Thacker, ${ }^{71}$ D. Boumediene,${ }^{34}$ C. Bourdarios, ${ }^{115}$ N. Bousson,${ }^{83}$ A. Boveia, ${ }^{31}$ J. Boyd,${ }^{30}$ I. R. Boyko, ${ }^{64}$ I. Bozovic-Jelisavcic,,${ }^{13 b}$ J. Bracinik, ${ }^{18}$ P. Branchini, ${ }^{134 a}$ A. Brandt, ${ }^{8}$ G. Brandt, ${ }^{118}$ O. Brandt, ${ }^{54}$ U. Bratzler, ${ }^{156}$ B. Brau, ${ }^{84}$ J. E. Brau, ${ }^{114}$ H. M. Braun, ${ }^{175, a}$ S. F. Brazzale, ${ }^{164 a, 164 c}$ B. Brelier, ${ }^{158}$ J. Bremer, ${ }^{30}$ K. Brendlinger, ${ }^{120}$ R. Brenner, ${ }^{166}$ S. Bressler, ${ }^{172}$ D. Britton, ${ }^{53}$ F. M. Brochu, ${ }^{28}$ I. Brock,${ }^{21}$ R. Brock,${ }^{88}$ F. Broggi, ${ }^{89 a}$ C. Bromberg,${ }^{88}$ J. Bronner, ${ }^{99}$ G. Brooijmans, ${ }^{35}$

T. Brooks, ${ }^{76}$ W. K. Brooks, ${ }^{32 b}$ G. Brown,${ }^{82}$ H. Brown, ${ }^{8}$ P. A. Bruckman de Renstrom, ${ }^{39}$ D. Bruncko, ${ }^{144 b}$

R. Bruneliere, ${ }^{48}$ S. Brunet, ${ }^{60}$ A. Bruni, ${ }^{20 a}$ G. Bruni, ${ }^{20 a}$ M. Bruschi,${ }^{20 a}$ T. Buanes, ${ }^{14}$ Q. Buat,${ }^{55}$ F. Bucci, ${ }^{49}$ J. Buchanan, ${ }^{118}$ P. Buchholz, ${ }^{141}$ R. M. Buckingham, ${ }^{118}$ A. G. Buckley, ${ }^{46}$ S. I. Buda ${ }^{26 a}$ I. A. Budagov, ${ }^{64}$ B. Budick, ${ }^{108}$ V. Büscher ${ }^{81}$ L. Bugge, ${ }^{117}$ O. Bulekov, ${ }^{96}$ A. C. Bundock, ${ }^{73}$ M. Bunse, ${ }^{43}$ T. Buran,${ }^{117}$ H. Burckhart, ${ }^{30}$ S. Burdin, ${ }^{73}$ T. Burgess, ${ }^{14}$ S. Burke, ${ }^{129}$ E. Busato,${ }^{34}$ P. Bussey,${ }^{53}$ C. P. Buszello, ${ }^{166}$ B. Butler, ${ }^{143}$ J. M. Butler,${ }^{22}$ C. M. Buttar, ${ }^{53}$ J. M. Butterworth, ${ }^{77}$ W. Buttinger, ${ }^{28}$ S. Cabrera Urbán, ${ }^{167}$ D. Caforio, ${ }^{20 a, 20 b}$ O. Cakir, ${ }^{4 a}$ P. Calafiura, ${ }^{15}$ G. Calderini, ${ }^{78}$

P. Calfayan, ${ }^{98}$ R. Calkins, ${ }^{106}$ L. P. Caloba, ${ }^{24 a}$ R. Caloi, ${ }^{132 a, 132 b}$ D. Calvet, ${ }^{34}$ S. Calvet, ${ }^{34}$ R. Camacho Toro, ${ }^{34}$ P. Camarri, ${ }^{133 a, 133 b}$ D. Cameron, ${ }^{117}$ L. M. Caminada, ${ }^{15}$ R. Caminal Armadans, ${ }^{12}$ S. Campana,${ }^{30}$ M. Campanelli, ${ }^{77}$ V. Canale, ${ }^{102 \mathrm{a}, 102 \mathrm{~b}}$ F. Canelli, ${ }^{31, \mathrm{~h}}$ A. Canepa, ${ }^{159 \mathrm{a}}$ J. Cantero, ${ }^{80}$ R. Cantrill, ${ }^{76}$ L. Capasso, ${ }^{102 \mathrm{a}, 102 \mathrm{~b}}$

M. D. M. Capeans Garrido, ${ }^{30}$ I. Caprini, ${ }^{26 a}$ M. Caprini, ${ }^{26 a}$ D. Capriotti, ${ }^{99}$ M. Capua,${ }^{37 a, 37 b}$ R. Caputo, ${ }^{81}$ R. Cardarelli, ${ }^{133 a}$ T. Carli ${ }^{30}$ G. Carlino, ${ }^{102 a}$ L. Carminati, ${ }^{89 a, 89 b}$ B. Caron, ${ }^{85}$ S. Caron, ${ }^{104}$ E. Carquin, ${ }^{32 b}$ G. D. Carrillo Montoya, ${ }^{173}$ A. A. Carter ${ }^{75}$ J. R. Carter,${ }^{28}$ J. Carvalho, ${ }^{124 a, i}$ D. Casadei, ${ }^{108}$ M. P. Casado, ${ }^{12}$ M. Cascella, ${ }^{122 a, 122 b}$ C. Caso, ${ }^{50 a, 50 b, a}$ A. M. Castaneda Hernandez, ${ }^{173, j}$ E. Castaneda-Miranda,${ }^{173}$

V. Castillo Gimenez, ${ }^{167}$ N. F. Castro, ${ }^{124 a}$ G. Cataldi, ${ }^{72 a}$ P. Catastini, ${ }^{57}$ A. Catinaccio, ${ }^{30}$ J. R. Catmore, ${ }^{30}$ A. Cattai,${ }^{30}$ G. Cattani, ${ }^{133 a, 133 b}$ S. Caughron, ${ }^{88}$ V. Cavaliere, ${ }^{165}$ P. Cavalleri, ${ }^{78}$ D. Cavalli, ${ }^{89 a}$ M. Cavalli-Sforza, ${ }^{12}$ V. Cavasinni, ${ }^{122 a, 122 b}$ F. Ceradini, ${ }^{134 a, 134 b}$ A. S. Cerqueira, ${ }^{24 b}$ A. Cerri, ${ }^{30}$ L. Cerrito, ${ }^{75}$ F. Cerutti, ${ }^{47}$ S. A. Cetin, ${ }^{19 b}$ A. Chafaq, ${ }^{135 a}$ D. Chakraborty, ${ }^{106}$ I. Chalupkova, ${ }^{126}$ K. Chan, ${ }^{3}$ P. Chang, ${ }^{165}$ B. Chapleau, ${ }^{85}$ J. D. Chapman, ${ }^{28}$ J. W. Chapman, ${ }^{87}$ E. Chareyre, ${ }^{78}$ D. G. Charlton, ${ }^{18}$ V. Chavda, ${ }^{82}$ C. A. Chavez Barajas, ${ }^{30}$ S. Cheatham,${ }^{85}$ S. Chekanov, ${ }^{6}$ S. V. Chekulaev, ${ }^{159 a}$ G. A. Chelkov, ${ }^{64}$ M. A. Chelstowska, ${ }^{104}$ C. Chen ${ }^{63}$ H. Chen ${ }^{25}$ S. Chen, ${ }^{33 c}$ X. Chen, ${ }^{173}$ Y. Chen, ${ }^{35}$ A. Cheplakov, ${ }^{64}$ R. Cherkaoui El Moursli, ${ }^{135 e}$ V. Chernyatin, ${ }^{25}$ E. Cheu, ${ }^{7}$ S. L. Cheung, ${ }^{158}$ L. Chevalier, ${ }^{136}$ G. Chiefari, ${ }^{102 a, 102 b}$ L. Chikovani,,${ }^{51 a, a}$ J. T. Childers,${ }^{30}$ A. Chilingarov, ${ }^{71}$ G. Chiodini, ${ }^{72 a}$ A. S. Chisholm, ${ }^{18}$ R. T. Chislett, ${ }^{77}$ A. Chitan, ${ }^{26 a}$ M. V. Chizhov, ${ }^{64}$ G. Choudalakis, ${ }^{31}$ S. Chouridou, ${ }^{137}$ I. A. Christidi, ${ }^{77}$ A. Christov, ${ }^{48}$ D. Chromek-Burckhart, ${ }^{30}$ M. L. Chu, ${ }^{151}$ J. Chudoba ${ }^{125}$ G. Ciapetti, ${ }^{132 a, 132 b}$ A. K. Ciftci, ${ }^{4 a}$ R. Ciftci, ${ }^{4 a}$ D. Cinca, ${ }^{34}$ V. Cindro, ${ }^{74}$ C. Ciocca, ${ }^{20 a, 20 b}$ A. Ciocio,,${ }^{15}$ M. Cirilli, ${ }^{87}$ P. Cirkovic, ${ }^{13 b}$ Z. H. Citron, ${ }^{172}$ M. Citterio, ${ }^{89 a}$ M. Ciubancan,${ }^{26 a}$ A. Clark,${ }^{49}$ P. J. Clark, ${ }^{46}$ R. N. Clarke, ${ }^{15}$ W. Cleland,${ }^{123}$ J. C. Clemens, ${ }^{83}$ B. Clement, ${ }^{55}$ C. Clement, ${ }^{146 a, 146 b}$ Y. Coadou, ${ }^{83}$ M. Cobal, ${ }^{164 a, 164 \mathrm{c}}$ A. Coccaro, ${ }^{138}$ J. Cochran, ${ }^{63}$ L. Coffey, ${ }^{23}$ J. G. Cogan, ${ }^{143}$ J. Coggeshall, ${ }^{165}$ E. Cogneras, ${ }^{178}$ J. Colas, ${ }^{5}$ S. Cole, ${ }^{106}$ A. P. Colijn, ${ }^{105}$ N. J. Collins, ${ }^{18}$ C. Collins-Tooth ${ }^{53}$ J. Collot, ${ }^{55}$ T. Colombo, ${ }^{119 a, 119 b}$ G. Colon, ${ }^{84}$ P. Conde Muiño, ${ }^{124 a}$ E. Coniavitis, ${ }^{118}$ M. C. Conidi, ${ }^{12}$ S. M. Consonni, ${ }^{89 a, 89 b}$ V. Consorti, ${ }^{48}$ S. Constantinescu, ${ }^{26 a}$ C. Conta, ${ }^{119 a, 119 b}$ G. Conti, ${ }^{57}$ F. Conventi, ${ }^{102 a, \mathrm{k}}$ M. Cooke, ${ }^{15}$ B. D. Cooper ${ }^{77}$ A. M. Cooper-Sarkar,${ }^{18}$ K. Copic, ${ }^{15}$ T. Cornelissen, ${ }^{175}$ M. Corradi,${ }^{20 a}$ F. Corriveau, ${ }^{85,1}$ A. Cortes-Gonzalez, ${ }^{165}$ G. Cortiana, ${ }^{99}$ G. Costa, ${ }^{89 a}$ M. J. Costa, ${ }^{167}$ D. Costanzo, ${ }^{139}$ D. Côté, ${ }^{30}$ L. Courneyea ${ }^{169}$ G. Cowan,${ }^{76}$ C. Cowden,${ }^{28}$ B. E. Cox,${ }^{82}$ K. Cranmer, ${ }^{108}$ F. Crescioli, ${ }^{122 a, 122 b}$ M. Cristinziani, ${ }^{21}$ G. Crosetti, ${ }^{37 a, 37 b}$ S. Crépé-Renaudin, ${ }^{55}$ C.-M. Cuciuc, ${ }^{26 a}$ C. Cuenca Almenar, ${ }^{176}$ T. Cuhadar Donszelmann, ${ }^{139}$ M. Curatolo, ${ }^{47}$ C. J. Curtis, ${ }^{18}$ C. Cuthbert, ${ }^{150}$ P. Cwetanski,${ }^{60}$ H. Czirr, ${ }^{141}$ P. Czodrowski, ${ }^{44}$ Z. Czyczula, ${ }^{176}$ S. D’Auria, ${ }^{53}$ M. D’Onofrio, ${ }^{73}$ A. D’Orazio, ${ }^{132 a, 132 b}$ M. J. Da Cunha Sargedas De Sousa, ${ }^{124 a}$ C. Da Via, ${ }^{82}$ W. Dabrowski, ${ }^{38}$ A. Dafinca, ${ }^{118}$ T. Dai, ${ }^{87}$ C. Dallapiccola,${ }^{84}$ M. Dam,${ }^{36}$ M. Dameri, ${ }^{50 a, 50 b}$ D. S. Damiani, ${ }^{137}$ H. O. Danielsson, ${ }^{30}$ V. Dao, ${ }^{49}$ G. Darbo, ${ }^{50 a}$ G. L. Darlea, ${ }^{26 b}$ J. A. Dassoulas, ${ }^{42}$ W. Davey, ${ }^{21}$ T. Davidek, ${ }^{126}$ N. Davidson, ${ }^{86}$ R. Davidson, ${ }^{71}$ E. Davies, ${ }^{118, \mathrm{~d}}$ M. Davies,${ }^{93}$ O. Davignon, ${ }^{78}$ A. R. Davison, ${ }^{77}$ Y. Davygora,${ }^{58 a}$ E. Dawe, ${ }^{142}$ I. Dawson, ${ }^{139}$ R. K. Daya-Ishmukhametova, ${ }^{23}$ K. De,${ }^{8}$ R. de Asmundis, ${ }^{102 a}$ S. De Castro, ${ }^{20 a, 20 b}$ S. De Cecco, ${ }^{78}$ J. de Graat, ${ }^{98}$ N. De Groot, ${ }^{104}$ P. de Jong, ${ }^{105}$ C. De La Taille, ${ }^{115}$ H. De la Torre,${ }^{80}$ F. De Lorenzi, ${ }^{63}$ L. de Mora, ${ }^{71}$ L. De Nooij, ${ }^{105}$ D. De Pedis, ${ }^{132 a}$ A. De Salvo, ${ }^{132 a}$ U. De Sanctis, ${ }^{164 a, 164 c}$ A. De Santo, ${ }^{149}$ J. B. De Vivie De Regie, ${ }^{115}$ G. De Zorzi,${ }^{132 a, 132 b}$ W. J. Dearnaley,${ }^{71}$ R. Debbe,${ }^{25}$ C. Debenedetti, ${ }^{46}$ B. Dechenaux, ${ }^{55}$ 
D. V. Dedovich, ${ }^{64}$ J. Degenhardt ${ }^{120}$ C. Del Papa,${ }^{164 a, 164 c}$ J. Del Peso, ${ }^{80}$ T. Del Prete, ${ }^{122 a, 122 b}$ T. Delemontex,${ }^{55}$ M. Deliyergiyev ${ }^{74}$ A. Dell'Acqua,${ }^{30}$ L. Dell'Asta ${ }^{22}$ M. Della Pietra,${ }^{102 a, k}$ D. della Volpe,${ }^{102 a, 102 b}$ M. Delmastro, ${ }^{5}$ P. A. Delsart, ${ }^{55}$ C. Deluca, ${ }^{105}$ S. Demers, ${ }^{176}$ M. Demichev, ${ }^{64}$ B. Demirkoz, ${ }^{12 m}$ J. Deng, ${ }^{163}$ S. P. Denisov, ${ }^{128}$ D. Derendarz, ${ }^{39}$ J.E. Derkaoui, ${ }^{135 d}$ F. Derue,${ }^{78}$ P. Dervan,${ }^{73}$ K. Desch, ${ }^{21}$ E. Devetak,${ }^{148}$ P. O. Deviveiros, ${ }^{105}$ A. Dewhurst ${ }^{129}$ B. DeWilde, ${ }^{148}$ S. Dhaliwal, ${ }^{158}$ R. Dhullipudi, ${ }^{25, n}$ A. Di Ciaccio, ${ }^{133 a, 133 b}$ L. Di Ciaccio, ${ }^{5}$ A. Di Girolamo, ${ }^{30}$ B. Di Girolamo, ${ }^{30}$ S. Di Luise, ${ }^{134 a, 134 b}$ A. Di Mattia,${ }^{173}$ B. Di Micco,${ }^{30}$ R. Di Nardo,${ }^{47}$ A. Di Simone, ${ }^{133 a, 133 b}$ R. Di Sipio, ${ }^{20 a, 20 b}$ M. A. Diaz, ${ }^{32 a}$ E. B. Diehl, ${ }^{87}$ J. Dietrich, ${ }^{42}$ T. A. Dietzsch, ${ }^{58 a}$ S. Diglio, ${ }^{86}$ K. Dindar Yagci, ${ }^{40}$ J. Dingfelder, ${ }^{21}$ F. Dinut, ${ }^{26 a}$ C. Dionisi, ${ }^{132 a, 132 b}$ P. Dita, ${ }^{26 a}$ S. Dita, ${ }^{26 a}$ F. Dittus, ${ }^{30}$ F. Djama ${ }^{83}$ T. Djobava, ${ }^{51 b}$ M. A. B. do Vale,${ }^{24 c}$ A. Do Valle Wemans, ${ }^{124 a, o}$ T. K. O. Doan, ${ }^{5}$ M. Dobbs,${ }^{85}$ R. Dobinson, ${ }^{30, a}$ D. Dobos,${ }^{30}$ E. Dobson,${ }^{30, p}$ J. Dodd,${ }^{35}$ C. Doglioni,${ }^{49}$ T. Doherty ${ }^{53}$ Y. Doi,${ }^{65, a}$ J. Dolejsi, ${ }^{126}$ I. Dolenc,${ }^{74}$ Z. Dolezal, ${ }^{126}$ B. A. Dolgoshein, ${ }^{96, a}$ T. Dohmae, ${ }^{155}$ M. Donadelli, ${ }^{24 \mathrm{~d}}$ J. Donini,${ }^{34}$ J. Dopke, ${ }^{30}$ A. Doria, ${ }^{102 a}$ A. Dos Anjos, ${ }^{173}$ A. Dotti, ${ }^{122 a, 122 b}$ M. T. Dova,${ }^{70}$ A. D. Doxiadis, ${ }^{105}$ A. T. Doyle,${ }^{53}$ M. Dris, ${ }^{10}$ J. Dubbert, ${ }^{99}$ S. Dube,${ }^{15}$ E. Duchovni, ${ }^{172}$ G. Duckeck, ${ }^{98}$ D. Duda,${ }^{175}$ A. Dudarev, ${ }^{30}$ F. Dudziak, ${ }^{63}$ M. Dührssen, ${ }^{30}$ I. P. Duerdoth, ${ }^{82}$ L. Duflot, ${ }^{115}$ M-A. Dufour,${ }^{85}$ L. Duguid, ${ }^{76}$ M. Dunford, ${ }^{30}$ H. Duran Yildiz, ${ }^{4 a}$ R. Duxfield, ${ }^{139}$ M. Dwuznik, ${ }^{38}$ F. Dydak,${ }^{30}$ M. Düren, ${ }^{52}$ J. Ebke, ${ }^{98}$ S. Eckweiler, ${ }^{81}$ K. Edmonds, ${ }^{81}$ W. Edson, ${ }^{2}$ C. A. Edwards, ${ }^{76}$ N. C. Edwards,${ }^{53}$ W. Ehrenfeld, ${ }^{42}$ T. Eifert, ${ }^{143}$ G. Eigen, ${ }^{14}$ K. Einsweiler, ${ }^{15}$ E. Eisenhandler, ${ }^{75}$ T. Ekelof, ${ }^{166}$ M. El Kacimi, ${ }^{135 c}$ M. Ellert, ${ }^{166}$ S. Elles, ${ }^{5}$ F. Ellinghaus, ${ }^{81}$ K. Ellis,${ }^{75}$ N. Ellis, ${ }^{30}$ J. Elmsheuser, ${ }^{98}$ M. Elsing, ${ }^{30}$ D. Emeliyanov, ${ }^{129}$ R. Engelmann, ${ }^{148}$ A. Engl, ${ }^{98}$ B. Epp,${ }^{61}$ J. Erdmann, ${ }^{54}$ A. Ereditato, ${ }^{17}$ D. Eriksson, ${ }^{146}$ J. Ernst, ${ }^{2}$ M. Ernst,${ }^{25}$ J. Ernwein, ${ }^{136}$ D. Errede, ${ }^{165}$ S. Errede, ${ }^{165}$ E. Ertel,${ }^{81}$ M. Escalier,${ }^{15}$ H. Esch,${ }^{43}$ C. Escobar, ${ }^{123}$ X. Espinal Curull, ${ }^{12}$ B. Esposito, ${ }^{47}$ F. Etienne, ${ }^{83}$ A. I. Etienvre, ${ }^{136}$ E. Etzion, ${ }^{153}$ D. Evangelakou,${ }^{54}$ H. Evans, ${ }^{60}$ L. Fabbri,${ }^{20 a, 20 b}$ C. Fabre, ${ }^{30}$ R. M. Fakhrutdinov, ${ }^{128}$ S. Falciano, ${ }^{132 a}$ Y. Fang, ${ }^{173}$ M. Fanti, ${ }^{89 a, 89 b}$ A. Farbin, ${ }^{8}$ A. Farilla, ${ }^{134 a}$ J. Farley, ${ }^{148}$ T. Farooque, ${ }^{158}$ S. Farrell, ${ }^{163}$ S. M. Farrington, ${ }^{170}$ P. Farthouat,${ }^{30}$ F. Fassi, ${ }^{167}$ P. Fassnacht,${ }^{30}$ D. Fassouliotis, ${ }^{9}$ B. Fatholahzadeh, ${ }^{158}$ A. Favareto, ${ }^{89 a, 89 b}$ L. Fayard, ${ }^{115}$ S. Fazio, ${ }^{37 a, 37 b}$ R. Febbraro, ${ }^{34}$ P. Federic,${ }^{144 a}$ O. L. Fedin, ${ }^{121}$ W. Fedorko ${ }^{88}$ M. Fehling-Kaschek ${ }^{48}$ L. Feligioni, ${ }^{83}$ D. Fellmann, ${ }^{6}$ C. Feng, ${ }^{33 d}$ E. J. Feng, ${ }^{6}$ A. B. Fenyuk, ${ }^{128}$ J. Ferencei, ${ }^{144 \mathrm{~b}}$ W. Fernando, ${ }^{6}$ S. Ferrag, ${ }^{53}$ J. Ferrando, ${ }^{53}$ V. Ferrara, ${ }^{42}$ A. Ferrari, ${ }^{166}$ P. Ferrari, ${ }^{105}$ R. Ferrari, ${ }^{119 a}$ D. E. Ferreira de Lima, ${ }^{53}$ A. Ferrer, ${ }^{167}$ D. Ferrere, ${ }^{49}$ C. Ferretti, ${ }^{87}$ A. Ferretto Parodi,,${ }^{50,50 b}$ M. Fiascaris, ${ }^{31}$ F. Fiedler, ${ }^{81}$ A. Filipčič,${ }^{74}$ F. Filthaut, ${ }^{104}$ M. Fincke-Keeler, ${ }^{169}$ M. C. N. Fiolhais,${ }^{124 a, i}$ L. Fiorini, ${ }^{167}$ A. Firan,${ }^{40}$ G. Fischer, ${ }^{42}$ M. J. Fisher, ${ }^{109}$ M. Flechl, ${ }^{48}$ I. Fleck, ${ }^{141}$ J. Fleckner, ${ }^{81}$ P. Fleischmann,,${ }^{174}$ S. Fleischmann, ${ }^{175}$ T. Flick, ${ }^{175}$ A. Floderus, ${ }^{79}$ L. R. Flores Castillo, ${ }^{173}$ M. J. Flowerdew, ${ }^{99}$ T. Fonseca Martin,${ }^{17}$ A. Formica, ${ }^{136}$ A. Forti, ${ }^{82}$ D. Fortin, ${ }^{159 a}$ D. Fournier, ${ }^{115}$ H. Fox,${ }^{71}$ P. Francavilla, ${ }^{12}$ M. Franchini, ${ }^{20 a, 20 b}$ S. Franchino, ${ }^{119 a, 119 b}$ D. Francis,${ }^{30}$ T. Frank, ${ }^{172}$ S. Franz,${ }^{30}$ M. Fraternali, ${ }^{119 a, 119 b}$ S. Fratina, ${ }^{120}$ S. T. French, ${ }^{28}$ C. Friedrich, ${ }^{42}$ F. Friedrich,${ }^{44}$ R. Froeschl,${ }^{30}$ D. Froidevaux, ${ }^{30}$ J. A. Frost,${ }^{28}$ C. Fukunaga,${ }^{156}$ E. Fullana Torregrosa, ${ }^{30}$ B. G. Fulsom, ${ }^{143}$ J. Fuster, ${ }^{167}$ C. Gabaldon, ${ }^{30}$ O. Gabizon, ${ }^{172}$ T. Gadfort, ${ }^{25}$ S. Gadomski, ${ }^{49}$ G. Gagliardi, ${ }^{50 a, 50 b}$ P. Gagnon, ${ }^{60}$ C. Galea, ${ }^{98}$ B. Galhardo, ${ }^{124 a}$ E. J. Gallas, ${ }^{118}$ V. Gallo, ${ }^{17}$ B. J. Gallop, ${ }^{129}$ P. Gallus, ${ }^{125}$ K. K. Gan, ${ }^{109}$ Y. S. Gao, ${ }^{143, f}$ A. Gaponenko, ${ }^{15}$ F. Garberson, ${ }^{176}$ M. Garcia-Sciveres, ${ }^{15}$ C. García,,${ }^{167}$ J. E. García Navarro, ${ }^{167}$ R. W. Gardner,${ }^{31}$ N. Garelli, ${ }^{30}$ H. Garitaonandia, ${ }^{105}$ V. Garonne, ${ }^{30}$ C. Gatti, ${ }^{47}$ G. Gaudio, ${ }^{119 a}$ B. Gaur, ${ }^{141}$ L. Gauthier, ${ }^{136}$ P. Gauzzi, ${ }^{132 a, 132 b}$ I. L. Gavrilenko, ${ }^{94}$ C. Gay, ${ }^{168}$ G. Gaycken,${ }^{21}$ E. N. Gazis, ${ }^{10}$ P. Ge,${ }^{33 d}$ Z. Gecse, ${ }^{168}$ C. N. P. Gee, ${ }^{129}$ D. A. A. Geerts, ${ }^{105}$ Ch. Geich-Gimbel, ${ }^{21}$ K. Gellerstedt, ${ }^{146 a, 146 b}$ C. Gemme, ${ }^{50 a}$ A. Gemmell,${ }^{53}$ M. H. Genest, ${ }^{55}$ S. Gentile, ${ }^{132 a, 132 b}$ M. George,${ }^{54}$ S. George,${ }^{76}$ P. Gerlach, ${ }^{175}$ A. Gershon, ${ }^{153}$ C. Geweniger,${ }^{58 a}$ H. Ghazlane,${ }^{135 b}$ N. Ghodbane,${ }^{34}$ B. Giacobbe, ${ }^{20 a}$ S. Giagu, ${ }^{132 a, 132 b}$ V. Giakoumopoulou, ${ }^{9}$ V. Giangiobbe, ${ }^{12}$ F. Gianotti, ${ }^{30}$ B. Gibbard ${ }^{25}$ A. Gibson, ${ }^{158}$ S. M. Gibson, ${ }^{30}$ D. Gillberg, ${ }^{29}$ A. R. Gillman, ${ }^{129}$ D. M. Gingrich,,${ }^{3, \mathrm{e}}$ J. Ginzburg, ${ }^{153}$ N. Giokaris, ${ }^{9}$ M. P. Giordani, ${ }^{164 \mathrm{c}}$ R. Giordano, ${ }^{102 \mathrm{a}, 102 \mathrm{~b}}$ F. M. Giorgi, ${ }^{16}$ P. Giovannini, ${ }^{99}$ P. F. Giraud, ${ }^{136}$ D. Giugni, ${ }^{89 \mathrm{a}}$ M. Giunta, ${ }^{93}$ P. Giusti, ${ }^{20 a}$ B. K. Gjelsten, ${ }^{117}$ L. K. Gladilin,,${ }^{97}$ C. Glasman, ${ }^{80}$ J. Glatzer, ${ }^{48}$ A. Glazov, ${ }^{42}$ K. W. Glitza, ${ }^{175}$

G. L. Glonti, ${ }^{64}$ J. R. Goddard, ${ }^{75}$ J. Godfrey, ${ }^{142}$ J. Godlewski, ${ }^{30}$ M. Goebel, ${ }^{42}$ T. Göpfert,${ }^{44}$ C. Goeringer, ${ }^{81}$

C. Gössling, ${ }^{43}$ S. Goldfarb, ${ }^{87}$ T. Golling, ${ }^{176}$ A. Gomes, ${ }^{124 a, c}$ L. S. Gomez Fajardo, ${ }^{42}$ R. Gonçalo, ${ }^{76}$

J. Goncalves Pinto Firmino Da Costa, ${ }^{42}$ L. Gonella,${ }^{21}$ S. González de la Hoz, ${ }^{167}$ G. Gonzalez Parra, ${ }^{12}$

M. L. Gonzalez Silva, ${ }^{27}$ S. Gonzalez-Sevilla, ${ }^{49}$ J. J. Goodson, ${ }^{148}$ L. Goossens, ${ }^{30}$ P. A. Gorbounov, ${ }^{95}$ H. A. Gordon, ${ }^{25}$ I. Gorelov, ${ }^{103}$ G. Gorfine, ${ }^{175}$ B. Gorini, ${ }^{30}$ E. Gorini, ${ }^{72 a, 72 b}$ A. Gorišek, ${ }^{74}$ E. Gornicki, ${ }^{39}$ B. Gosdzik, ${ }^{42}$ A. T. Goshaw, ${ }^{6}$ M. Gosselink, ${ }^{105}$ M. I. Gostkin, ${ }^{64}$ I. Gough Eschrich, ${ }^{163}$ M. Gouighri, ${ }^{135 a}$ D. Goujdami, ${ }^{135 c}$ M. P. Goulette, ${ }^{49}$

A. G. Goussiou, ${ }^{138}$ C. Goy, ${ }^{5}$ S. Gozpinar, ${ }^{23}$ I. Grabowska-Bold, ${ }^{38}$ P. Grafström, ${ }^{20 a, 20 b}$ K-J. Grahn, ${ }^{42}$ F. Grancagnolo, ${ }^{72 a}$ S. Grancagnolo, ${ }^{16}$ V. Grassi, ${ }^{148}$ V. Gratchev, ${ }^{121}$ N. Grau, ${ }^{35}$ H. M. Gray, ${ }^{30}$ J. A. Gray, ${ }^{148}$ 
E. Graziani, ${ }^{134 a}$ O. G. Grebenyuk, ${ }^{121}$ T. Greenshaw, ${ }^{73}$ Z. D. Greenwood, ${ }^{25, n}$ K. Gregersen, ${ }^{36}$ I. M. Gregor, ${ }^{42}$ P. Grenier, ${ }^{143}$ J. Griffiths, ${ }^{8}$ N. Grigalashvili, ${ }^{64}$ A. A. Grillo, ${ }^{137}$ S. Grinstein, ${ }^{12} \mathrm{Ph}$. Gris, ${ }^{34}$ Y. V. Grishkevich, ${ }^{97}$ J.-F. Grivaz, ${ }^{115}$ E. Gross, ${ }^{172}$ J. Grosse-Knetter, ${ }^{54}$ J. Groth-Jensen, ${ }^{172}$ K. Grybel,,${ }^{141}$ D. Guest,,${ }^{176}$ C. Guicheney, ${ }^{34}$

S. Guindon, ${ }^{54}$ U. Gul, ${ }^{53}$ H. Guler, ${ }^{85, q}$ J. Gunther, ${ }^{125}$ B. Guo, ${ }^{158}$ J. Guo, ${ }^{35}$ P. Gutierrez, ${ }^{111}$ N. Guttman, ${ }^{153}$ O. Gutzwiller, ${ }^{173}$ C. Guyot, ${ }^{136}$ C. Gwenlan, ${ }^{18}$ C. B. Gwilliam, ${ }^{73}$ A. Haas, ${ }^{143}$ S. Haas, ${ }^{30}$ C. Haber, ${ }^{15}$ H. K. Hadavand, ${ }^{40}$ D. R. Hadley, ${ }^{18}$ P. Haefner, ${ }^{21}$ F. Hahn, ${ }^{30}$ S. Haider,${ }^{30}$ Z. Hajduk, ${ }^{39}$ H. Hakobyan, ${ }^{177}$ D. Hall, ${ }^{118}$ J. Haller, ${ }^{54}$ K. Hamacher, ${ }^{175}$ P. Hamal, ${ }^{113}$ M. Hamer, ${ }^{54}$ A. Hamilton, ${ }^{145 b, r}$ S. Hamilton, ${ }^{161}$ L. Han, ${ }^{33 b}$ K. Hanagaki, ${ }^{116}$ K. Hanawa, ${ }^{160}$ M. Hance, ${ }^{15}$ C. Handel, ${ }^{81}$ P. Hanke, ${ }^{58 a}$ J. R. Hansen, ${ }^{36}$ J. B. Hansen, ${ }^{36}$ J. D. Hansen, ${ }^{36}$ P. H. Hansen, ${ }^{36}$ P. Hansson, ${ }^{143}$ K. Hara, ${ }^{160}$ G. A. Hare, ${ }^{137}$ T. Harenberg, ${ }^{175}$ S. Harkusha, ${ }^{90}$ D. Harper, ${ }^{87}$ R. D. Harrington, ${ }^{46}$ O. M. Harris, ${ }^{138}$ J. Hartert, ${ }^{48}$ F. Hartjes, ${ }^{105}$ T. Haruyama, ${ }^{65}$ A. Harvey, ${ }^{56}$ S. Hasegawa, ${ }^{101}$ Y. Hasegawa, ${ }^{140}$

S. Hassani, ${ }^{136}$ S. Haug, ${ }^{17}$ M. Hauschild, ${ }^{30}$ R. Hauser, ${ }^{88}$ M. Havranek,${ }^{21}$ C. M. Hawkes, ${ }^{18}$ R. J. Hawkings,${ }^{30}$ A. D. Hawkins, ${ }^{79}$ T. Hayakawa, ${ }^{66}$ T. Hayashi, ${ }^{160}$ D. Hayden, ${ }^{76}$ C. P. Hays, ${ }^{18}$ H. S. Hayward,${ }^{73}$ S. J. Haywood, ${ }^{129}$ S. J. Head,${ }^{18}$ V. Hedberg, ${ }^{79}$ L. Heelan, ${ }^{8}$ S. Heim,${ }^{88}$ B. Heinemann, ${ }^{15}$ S. Heisterkamp, ${ }^{36}$ L. Helary, ${ }^{22}$ C. Heller, ${ }^{98}$ M. Heller ${ }^{30}$ S. Hellman, ${ }^{146 a, 146 b}$ D. Hellmich, ${ }^{21}$ C. Helsens, ${ }^{12}$ R. C. W. Henderson, ${ }^{71}$ M. Henke, ${ }^{58 a}$ A. Henrichs, ${ }^{54}$ A. M. Henriques Correia, ${ }^{30}$ S. Henrot-Versille, ${ }^{115}$ C. Hensel,${ }^{54}$ T. Henß, ${ }^{175}$ C. M. Hernandez, ${ }^{8}$

Y. Hernández Jiménez, ${ }^{167}$ R. Herrberg, ${ }^{16}$ G. Herten, ${ }^{48}$ R. Hertenberger ${ }^{98}$ L. Hervas, ${ }^{30}$ G. G. Hesketh,${ }^{77}$ N.P. Hessey, ${ }^{105}$ E. Higón-Rodriguez, ${ }^{167}$ J. C. Hill, ${ }^{28}$ K. H. Hiller, ${ }^{42}$ S. Hillert, ${ }^{21}$ S. J. Hillier, ${ }^{18}$ I. Hinchliffe, ${ }^{15}$

E. Hines, ${ }^{120}$ M. Hirose,${ }^{116}$ F. Hirsch,${ }^{43}$ D. Hirschbuehl, ${ }^{175}$ J. Hobbs, ${ }^{148}$ N. Hod, ${ }^{153}$ M. C. Hodgkinson, ${ }^{139}$

P. Hodgson, ${ }^{139}$ A. Hoecker, ${ }^{30}$ M. R. Hoeferkamp, ${ }^{103}$ J. Hoffman, ${ }^{40}$ D. Hoffmann,${ }^{83}$ M. Hohlfeld,${ }^{81}$ M. Holder, ${ }^{141}$

S. O. Holmgren, ${ }^{146 a}$ T. Holy, ${ }^{127}$ J. L. Holzbauer, ${ }^{88}$ T. M. Hong, ${ }^{120}$ L. Hooft van Huysduynen, ${ }^{108}$ S. Horner, ${ }^{48}$ J-Y. Hostachy, ${ }^{55}$ S. Hou ${ }^{151}$ A. Hoummada, ${ }^{135 a}$ J. Howard, ${ }^{118}$ J. Howarth, ${ }^{82}$ I. Hristova, ${ }^{16}$ J. Hrivnac, ${ }^{115}$ T. Hryn' ova, P. J. Hsu, ${ }^{81}$ S.-C. Hsu, ${ }^{15}$ D. Hu ${ }^{35}$ Z. Hubacek, ${ }^{127}$ F. Hubaut,${ }^{83}$ F. Huegging, ${ }^{21}$ A. Huettmann, ${ }^{42}$ T. B. Huffman, ${ }^{118}$ E. W. Hughes,${ }^{35}$ G. Hughes,${ }^{71}$ M. Huhtinen,${ }^{30}$ M. Hurwitz, ${ }^{15}$ U. Husemann, ${ }^{42}$ N. Huseynov, ${ }^{64, s}$ J. Huston,,${ }^{88}$ J. Huth, ${ }^{57}$ G. Iacobucci, ${ }^{49} \mathrm{G}$. Iakovidis, ${ }^{10} \mathrm{M}$. Ibbotson, ${ }^{82}$ I. Ibragimov, ${ }^{141} \mathrm{~L}$. Iconomidou-Fayard ${ }^{115} \mathrm{~J}$. Idarraga, ${ }^{115} \mathrm{P}$. Iengo, ${ }^{102 \mathrm{a}}$ O. Igonkina, ${ }^{105}$ Y. Ikegami, ${ }^{65}$ M. Ikeno,${ }^{65}$ D. Iliadis, ${ }^{154} \mathrm{~N}$. Ilic, ${ }^{158}$ T. Ince, ${ }^{21}$ J. Inigo-Golfin, ${ }^{30}$ P. Ioannou, ${ }^{9}$ M. Iodice, ${ }^{134 \mathrm{a}} \mathrm{K}$. Iordanidou, ${ }^{9}$ V. Ippolito, ${ }^{132 \mathrm{a}, 132 \mathrm{~b}}$ A. Irles Quiles, ${ }^{167} \mathrm{C}$. Isaksson, ${ }^{166} \mathrm{M}$. Ishino, ${ }^{67} \mathrm{M}$. Ishitsuka, ${ }^{157}$ R. Ishmukhametov, ${ }^{40} \mathrm{C}$. Issever, ${ }^{18} \mathrm{~S}$. Istin, ${ }^{19 \mathrm{a}} \mathrm{A}$. V. Ivashin, ${ }^{128} \mathrm{~W}$. Iwanski, ${ }^{39} \mathrm{H}$. Iwasaki, ${ }^{65} \mathrm{~J}$. M. Izen, ${ }^{41} \mathrm{~V}$. Izzo, ${ }^{102 \mathrm{a}}$ B. Jackson, ${ }^{120}$ J. N. Jackson, ${ }^{73}$ P. Jackson, ${ }^{1}$ M. R. Jaekel,${ }^{30}$ V. Jain, ${ }^{60}$ K. Jakobs, ${ }^{48}$ S. Jakobsen, ${ }^{36}$ T. Jakoubek, ${ }^{125}$ J. Jakubek, ${ }^{127}$ D. K. Jana, ${ }^{111}$ E. Jansen, ${ }^{77}$ H. Jansen, ${ }^{30}$ A. Jantsch,${ }^{99}$ M. Janus, ${ }^{48}$ G. Jarlskog, ${ }^{79}$ L. Jeanty, ${ }^{57}$ I. Jen-La Plante, ${ }^{31}$ D. Jennens, ${ }^{86}$ P. Jenni, ${ }^{30}$ A. E. Loevschall-Jensen, ${ }^{36}$ P. Jež,${ }^{36}$ S. Jézéquel, ${ }^{5}$ M. K. Jha, ${ }^{20 a}$ H. Ji, ${ }^{173}$ W. Ji, ${ }^{81}$ J. Jia, ${ }^{148}$ Y. Jiang, ${ }^{33 b}$ M. Jimenez Belenguer, ${ }^{42}$ S. Jin,${ }^{33 a}$ O. Jinnouchi, ${ }^{157}$ M. D. Joergensen, ${ }^{36}$ D. Joffe, ${ }^{40}$ M. Johansen, ${ }^{146 a, 146 b}$ K. E. Johansson, ${ }^{146 a}$ P. Johansson, ${ }^{139}$ S. Johnert, ${ }^{42}$ K. A. Johns, ${ }^{7}$ K. Jon-And, ${ }^{146 a, 146 b}$ G. Jones, ${ }^{170}$ R. W. L. Jones, ${ }^{71}$ T. J. Jones,${ }^{73}$ C. Joram, ${ }^{30}$ P. M. Jorge, ${ }^{124 a}$ K. D. Joshi, ${ }^{82}$ J. Jovicevic, ${ }^{147}$ T. Jovin, ${ }^{13 b}$ X. Ju, ${ }^{173}$ C. A. Jung, ${ }^{43}$ R. M. Jungst,${ }^{30}$ V. Juranek, ${ }^{125}$ P. Jussel, ${ }^{61}$ A. Juste Rozas, ${ }^{12}$ S. Kabana, ${ }^{17}$ M. Kaci,${ }^{167}$ A. Kaczmarska, ${ }^{39}$ P. Kadlecik, ${ }^{36}$ M. Kado, ${ }^{115}$ H. Kagan, ${ }^{109}$ M. Kagan, ${ }^{57}$ E. Kajomovitz, ${ }^{152}$ S. Kalinin, ${ }^{175}$ L. V. Kalinovskaya, ${ }^{64}$ S. Kama, ${ }^{40}$ N. Kanaya, ${ }^{155}$ M. Kaneda, ${ }^{30}$ S. Kaneti, ${ }^{28}$ T. Kanno, ${ }^{157}$ V. A. Kantserov, ${ }^{96}$ J. Kanzaki, ${ }^{65}$ B. Kaplan, ${ }^{108}$ A. Kapliy, ${ }^{31}$ J. Kaplon, ${ }^{30}$ D. Kar, ${ }^{53}$ M. Karagounis,${ }^{21}$ K. Karakostas, ${ }^{10}$ M. Karnevskiy, ${ }^{42}$ V. Kartvelishvili, ${ }^{71}$ A. N. Karyukhin, ${ }^{128}$ L. Kashif, ${ }^{173}$ G. Kasieczka, ${ }^{58 b}$ R. D. Kass, ${ }^{109}$ A. Kastanas, ${ }^{14}$ M. Kataoka, ${ }^{5}$ Y. Kataoka, ${ }^{155}$ E. Katsoufis,${ }^{10}$ J. Katzy, ${ }^{42}$ V. Kaushik, ${ }^{7}$ K. Kawagoe,${ }^{69}$ T. Kawamoto, ${ }^{155}$ G. Kawamura,${ }^{81}$ M. S. Kayl, ${ }^{105}$ S. Kazama, ${ }^{155}$ V. A. Kazanin, ${ }^{107}$ M. Y. Kazarinov, ${ }^{64}$ R. Keeler, ${ }^{169}$ R. Kehoe, ${ }^{40}$ M. Keil,${ }^{54}$ G. D. Kekelidze, ${ }^{64}$ J. S. Keller, ${ }^{138}$ M. Kenyon,${ }^{53}$ O. Kepka, ${ }^{125}$ N. Kerschen,${ }^{30}$ B. P. Kerševan, ${ }^{74}$ S. Kersten, ${ }^{175}$ K. Kessoku, ${ }^{155}$ J. Keung, ${ }^{158}$ F. Khalil-zada, ${ }^{11}$ H. Khandanyan, ${ }^{146 a, 146 b}$ A. Khanov, ${ }^{112}$ D. Kharchenko, ${ }^{64}$ A. Khodinov, ${ }^{96}$ A. Khomich, ${ }^{58 a}$ T. J. Khoo, ${ }^{28}$ G. Khoriauli, ${ }^{21}$ A. Khoroshilov, ${ }^{175}$ V. Khovanskiy, ${ }^{95}$ E. Khramov, ${ }^{64}$ J. Khubua, ${ }^{51 \mathrm{~b}}$ H. Kim, ${ }^{146 a, 146 \mathrm{~b}}$ S. H. Kim, ${ }^{160}$ N. Kimura, ${ }^{171}$ O. Kind, ${ }^{16}$ B. T. King, ${ }^{73}$ M. King, ${ }^{66}$ R. S. B. King, ${ }^{118}$ J. Kirk, ${ }^{129}$ A. E. Kiryunin, ${ }^{99}$ T. Kishimoto,${ }^{66}$ D. Kisielewska, ${ }^{38}$ T. Kitamura, ${ }^{66}$ T. Kittelmann, ${ }^{123}$ K. Kiuchi, ${ }^{160}$ E. Kladiva, ${ }^{144 \mathrm{~b}}$ M. Klein, ${ }^{73}$ U. Klein, ${ }^{73}$ K. Kleinknecht, ${ }^{81}$ M. Klemetti, ${ }^{85}$ A. Klier, ${ }^{172}$ P. Klimek, ${ }^{146 a, 146 b}$ A. Klimentov, ${ }^{25}$ R. Klingenberg, ${ }^{43}$ J. A. Klinger, ${ }^{82}$ E. B. Klinkby, ${ }^{36}$ T. Klioutchnikova, ${ }^{30}$ P. F. Klok, ${ }^{104}$ S. Klous, ${ }^{105}$ E.-E. Kluge, ${ }^{58 a}$ T. Kluge, ${ }^{73}$ P. Kluit, ${ }^{105}$ S. Kluth, ${ }^{99}$ N. S. Knecht, ${ }^{158}$ E. Kneringer,${ }^{61}$ E. B. F. G. Knoops ${ }^{83}$ A. Knue ${ }^{54}$ B. R. Ko, ${ }^{45}$ T. Kobayashi, ${ }^{155}$ M. Kobel, ${ }^{44}$ M. Kocian, ${ }^{143}$ P. Kodys, ${ }^{126}$ K. Köneke, ${ }^{30}$ A. C. König, ${ }^{104}$ S. Koenig, ${ }^{81}$ L. Köpke, ${ }^{81}$ F. Koetsveld, ${ }^{104}$ P. Koevesarki, ${ }^{21}$ T. Koffas, ${ }^{29}$ E. Koffeman, ${ }^{105}$ L. A. Kogan, ${ }^{18}$ S. Kohlmann, ${ }^{175}$ F. Kohn, ${ }^{54}$ Z. Kohout, ${ }^{127}$ T. Kohriki, ${ }^{65}$ T. Koi, ${ }^{143}$ G. M. Kolachev, ${ }^{107, a}$ H. Kolanoski, ${ }^{16}$ V. Kolesnikov, ${ }^{64}$ 
I. Koletsou, ${ }^{89 \mathrm{a}}$ J. Koll, ${ }^{88}$ A. A. Komar, ${ }^{94}$ Y. Komori, ${ }^{155}$ T. Kondo, ${ }^{65}$ T. Kono, ${ }^{42, t}$ A. I. Kononov, ${ }^{48}$ R. Konoplich, ${ }^{108, u}$ N. Konstantinidis, ${ }^{77}$ S. Koperny,${ }^{38}$ K. Korcyl,${ }^{39}$ K. Kordas, ${ }^{154}$ A. Korn, ${ }^{118}$ A. Korol, ${ }^{107}$ I. Korolkov, ${ }^{12}$ E. V. Korolkova, ${ }^{139}$ V. A. Korotkov, ${ }^{128}$ O. Kortner, ${ }^{99}$ S. Kortner, ${ }^{99}$ V. V. Kostyukhin, ${ }^{21}$ S. Kotov, ${ }^{99}$ V. M. Kotov, ${ }^{64}$ A. Kotwal, ${ }^{45}$ C. Kourkoumelis, ${ }^{9}$ V. Kouskoura, ${ }^{154}$ A. Koutsman, ${ }^{159 a}$ R. Kowalewski, ${ }^{169}$ T.Z. Kowalski, ${ }^{38}$ W. Kozanecki, ${ }^{136}$ A. S. Kozhin, ${ }^{128}$ V. Kral, ${ }^{127}$ V. A. Kramarenko, ${ }^{97}$ G. Kramberger ${ }^{74}$ M. W. Krasny, ${ }^{78}$ A. Krasznahorkay, ${ }^{108}$ J. K. Kraus, ${ }^{21}$ S. Kreiss, ${ }^{108}$ F. Krejci, ${ }^{127}$ J. Kretzschmar, ${ }^{73}$ N. Krieger, ${ }^{54}$ P. Krieger, ${ }^{158}$ K. Kroeninger, ${ }^{54}$ H. Kroha, ${ }^{99}$ J. Kroll,,${ }^{120}$ J. Kroseberg, ${ }^{21}$ J. Krstic, ${ }^{13 a}$ U. Kruchonak, ${ }^{64}$ H. Krüger, ${ }^{21}$ T. Kruker, ${ }^{17}$ N. Krumnack, ${ }^{63}$ Z. V. Krumshteyn, ${ }^{64}$ T. Kubota, ${ }^{86}$ S. Kuday ${ }^{4 a}$ S. Kuehn, ${ }^{48}$ A. Kugel,${ }^{58 c}$ T. Kuhl,${ }^{42}$ D. Kuhn, ${ }^{61}$ V. Kukhtin, ${ }^{64}$ Y. Kulchitsky, ${ }^{90}$ S. Kuleshov ${ }^{32 b}$ C. Kummer,${ }^{98}$ M. Kuna, ${ }^{78}$ J. Kunkle, ${ }^{120}$ A. Kupco, ${ }^{125}$ H. Kurashige, ${ }^{66}$ M. Kurata, ${ }^{160}$ Y. A. Kurochkin, ${ }^{90}$ V. Kus, ${ }^{125}$ E. S. Kuwertz, ${ }^{147}$ M. Kuze, ${ }^{157}$ J. Kvita, ${ }^{142}$ R. Kwee, ${ }^{16}$ A. La Rosa ${ }^{49}$ L. La Rotonda, ${ }^{37 a, 37 b}$ L. Labarga ${ }^{80}$ J. Labbe, ${ }^{5}$ S. Lablak, ${ }^{135 a}$ C. Lacasta, ${ }^{167}$ F. Lacava, ${ }^{132 a, 132 b}$ H. Lacker, ${ }^{16}$ D. Lacour,${ }^{78}$ V. R. Lacuesta, ${ }^{167}$ E. Ladygin, ${ }^{64}$ R. Lafaye, ${ }^{5}$ B. Laforge,${ }^{78}$ T. Lagouri, ${ }^{176}$ S. Lai, ${ }^{48}$ E. Laisne, ${ }^{55}$ M. Lamanna, ${ }^{30}$ L. Lambourne, ${ }^{77}$ C. L. Lampen, ${ }^{7}$ W. Lampl, ${ }^{7}$ E. Lancon, ${ }^{136}$ U. Landgraf, ${ }^{48}$ M. P. J. Landon,${ }^{75}$ J. L. Lane, ${ }^{82}$ V. S. Lang, ${ }^{58 a}$ C. Lange,${ }^{42}$ A. J. Lankford, ${ }^{163}$ F. Lanni, ${ }^{25}$ K. Lantzsch, ${ }^{175}$ S. Laplace, ${ }^{78}$ C. Lapoire, ${ }^{21}$ J. F. Laporte, ${ }^{136}$ T. Lari, ${ }^{89 a}$ A. Larner, ${ }^{118}$ M. Lassnig, ${ }^{30}$ P. Laurelli, ${ }^{47}$ V. Lavorini,,${ }^{37,37 b}$ W. Lavrijsen, ${ }^{15}$ P. Laycock,${ }^{73}$ O. Le Dortz, ${ }^{78}$ E. Le Guirriec, ${ }^{83}$ E. Le Menedeu, ${ }^{12}$ T. LeCompte, ${ }^{6}$ F. Ledroit-Guillon, ${ }^{55}$ H. Lee, ${ }^{105}$ J. S. H. Lee, ${ }^{116}$ S. C. Lee,${ }^{151}$ L. Lee, ${ }^{176}$ M. Lefebvre, ${ }^{169}$ M. Legendre, ${ }^{136}$ F. Legger, ${ }^{98}$ C. Leggett,,${ }^{15}$ M. Lehmacher, ${ }^{21}$ G. Lehmann Miotto, ${ }^{30}$ X. Lei, ${ }^{7}$ M. A. L. Leite, ${ }^{24 d}$ R. Leitner, ${ }^{126}$ D. Lellouch, ${ }^{172}$ B. Lemmer, ${ }^{54}$ V. Lendermann, ${ }^{58 a}$ K. J. C. Leney, ${ }^{145 b}$ T. Lenz, ${ }^{105}$ G. Lenzen, ${ }^{175}$ B. Lenzi, ${ }^{30}$ K. Leonhardt, ${ }^{44}$ S. Leontsinis, ${ }^{10}$ F. Lepold,${ }^{58 a}$ C. Leroy, ${ }^{93}$ J-R. Lessard, ${ }^{169}$ C. G. Lester ${ }^{28}$ C. M. Lester, ${ }^{120}$ J. Levêque,${ }^{5}$ D. Levin, ${ }^{87}$ L. J. Levinson, ${ }^{172}$ A. Lewis, ${ }^{118}$ G. H. Lewis, ${ }^{108}$ A. M. Leyko, ${ }^{21}$ M. Leyton, ${ }^{16}$ B. Li ${ }^{83} \mathrm{H} . \mathrm{Li},{ }^{173, v} \mathrm{~S}$. Li $,{ }^{33 b, w} \mathrm{X} . \mathrm{Li},{ }^{87}$ Z. Liang, ${ }^{118, \mathrm{x}} \mathrm{H} . \mathrm{Liao},{ }^{34}$ B. Liberti, ${ }^{133 a}$ P. Lichard,${ }^{30}$ M. Lichtnecker ${ }^{98} \mathrm{~K}$. Lie, ${ }^{165}$ W. Liebig,,${ }^{14}$ C. Limbach,${ }^{21}$ A. Limosani, ${ }^{86}$ M. Limper, ${ }^{62}$ S. C. Lin, ${ }^{151, y}$ F. Linde, ${ }^{105}$ J. T. Linnemann, ${ }^{88}$ E. Lipeles, ${ }^{120}$ A. Lipniacka, ${ }^{14}$ T. M. Liss, ${ }^{165}$ D. Lissauer, ${ }^{25}$ A. Lister ${ }^{49}$ A. M. Litke, ${ }^{137}$ C. Liu, ${ }^{29}$ D. Liu, ${ }^{151}$ H. Liu, ${ }^{87}$ J. B. Liu, ${ }^{87}$ L. Liu, ${ }^{87}$ M. Liu, ${ }^{33 b}$ Y. Liu, ${ }^{33 b}$ M. Livan, ${ }^{119 a, 119 b}$ S. S. A. Livermore, ${ }^{118}$ A. Lleres, ${ }^{55}$ J. Llorente Merino, ${ }^{80}$ S. L. Lloyd, ${ }^{75}$ E. Lobodzinska, ${ }^{42}$ P. Loch, ${ }^{7}$ W. S. Lockman, ${ }^{137}$ T. Loddenkoetter, ${ }^{21}$ F. K. Loebinger, ${ }^{82}$ A. Loginov, ${ }^{176}$ C. W. Loh, ${ }^{168}$ T. Lohse, ${ }^{16}$ K. Lohwasser, ${ }^{48}$ M. Lokajicek, ${ }^{125}$ V. P. Lombardo, ${ }^{5}$ R. E. Long, ${ }^{71}$ L. Lopes,${ }^{124 a}$ D. Lopez Mateos,${ }^{57}$ J. Lorenz, ${ }^{98}$

N. Lorenzo Martinez, ${ }^{115}$ M. Losada, ${ }^{162}$ P. Loscutoff, ${ }^{15}$ F. Lo Sterzo, ${ }^{132 a, 132 b}$ M. J. Losty,${ }^{159 a, a}$ X. Lou, ${ }^{41}$ A. Lounis, ${ }^{115}$ K. F. Loureiro, ${ }^{162}$ J. Love,${ }^{6}$ P. A. Love, ${ }^{71}$ A. J. Lowe, ${ }^{143, f}$ F. Lu, ${ }^{33 a}$ H. J. Lubatti, ${ }^{138}$ C. Luci, ${ }^{132 a, 132 b}$ A. Lucotte ${ }^{55}$ A. Ludwig, ${ }^{44}$ D. Ludwig, ${ }^{42}$ I. Ludwig, ${ }^{48}$ J. Ludwig, ${ }^{48}$ F. Luehring, ${ }^{60}$ G. Luijckx,${ }^{105}$ W. Lukas, ${ }^{61}$ D. Lumb,${ }^{48}$ L. Luminari, ${ }^{132 \mathrm{a}}$ E. Lund, ${ }^{117}$ B. Lund-Jensen, ${ }^{147}$ B. Lundberg ${ }^{79}$ J. Lundberg, ${ }^{146 a, 146 \mathrm{~b}}$ O. Lundberg, ${ }^{146 a, 146 \mathrm{~b}}$ J. Lundquist,${ }^{36}$ M. Lungwitz, ${ }^{81}$ D. Lynn, ${ }^{25}$ E. Lytken, ${ }^{79}$ H. Ma,${ }^{25}$ L. L. Ma,${ }^{173}$ G. Maccarrone, ${ }^{47}$ A. Macchiolo, ${ }^{99}$ B. Maček, ${ }^{74}$ J. Machado Miguens, ${ }^{124 a}$ R. Mackeprang, ${ }^{36}$ R. J. Madaras, ${ }^{15}$ H. J. Maddocks, ${ }^{71}$ W. F. Mader,${ }^{44}$ R. Maenner ${ }^{58 c}$ T. Maeno, ${ }^{25}$ P. Mättig, ${ }^{175}$ S. Mättig, ${ }^{81}$ L. Magnoni, ${ }^{163}$ E. Magradze,${ }^{54}$ K. Mahboubi, ${ }^{48}$ J. Mahlstedt, ${ }^{105}$ S. Mahmoud, ${ }^{73}$ G. Mahout, ${ }^{18}$ C. Maiani, ${ }^{136}$ C. Maidantchik, ${ }^{24 a}$ A. Maio, ${ }^{124 a, c}$ S. Majewski, ${ }^{25}$ Y. Makida, ${ }^{65}$ N. Makovec, ${ }^{115}$ P. Mal, ${ }^{136}$ B. Malaescu, ${ }^{30}$ Pa. Malecki, ${ }^{39}$ P. Malecki, ${ }^{39}$ V. P. Maleev, ${ }^{121}$ F. Malek, ${ }^{55}$

U. Mallik, ${ }^{62}$ D. Malon, ${ }^{6}$ C. Malone,${ }^{143}$ S. Maltezos, ${ }^{10}$ V. Malyshev,${ }^{107}$ S. Malyukov, ${ }^{30}$ R. Mameghani,${ }^{98}$ J. Mamuzic, ${ }^{13 b}$ A. Manabe, ${ }^{65}$ L. Mandelli, ${ }^{89 a}$ I. Mandić, ${ }^{74}$ R. Mandrysch,,${ }^{16}$ J. Maneira, ${ }^{124 a}$ A. Manfredini, ${ }^{99}$ P. S. Mangeard ${ }^{88}$ L. Manhaes de Andrade Filho, ${ }^{24 \mathrm{~b}}$ J. A. Manjarres Ramos, ${ }^{136}$ A. Mann, ${ }^{54}$ P. M. Manning, ${ }^{137}$

A. Manousakis-Katsikakis, ${ }^{9}$ B. Mansoulie, ${ }^{136}$ A. Mapelli, ${ }^{30}$ L. Mapelli ${ }^{30}$ L. March, ${ }^{80}$ J. F. Marchand,${ }^{29}$ F. Marchese, ${ }^{133 a, 133 b}$ G. Marchiori, ${ }^{78}$ M. Marcisovsky, ${ }^{125}$ C. P. Marino, ${ }^{169}$ F. Marroquim, ${ }^{24 a}$ Z. Marshall, ${ }^{30}$ F. K. Martens, ${ }^{158}$ L. F. Marti, ${ }^{17}$ S. Marti-Garcia, ${ }^{167}$ B. Martin, ${ }^{30}$ B. Martin, ${ }^{88}$ J. P. Martin, ${ }^{93}$ T. A. Martin, ${ }^{18}$ V. J. Martin, ${ }^{46}$ B. Martin dit Latour, ${ }^{49}$ S. Martin-Haugh, ${ }^{149}$ M. Martinez, ${ }^{12}$ V. Martinez Outschoorn, ${ }^{57}$ A. C. Martyniuk, ${ }^{169}$ M. Marx,${ }^{82}$ F. Marzano, ${ }^{132 a}$ A. Marzin, ${ }^{111}$ L. Masetti, ${ }^{81}$ T. Mashimo, ${ }^{155}$ R. Mashinistov,${ }^{94}$ J. Masik, ${ }^{82}$ A. L. Maslennikov, ${ }^{107}$ I. Massa, ${ }^{20 a, 20 b}$ G. Massaro, ${ }^{105}$ N. Massol, ${ }^{5}$ P. Mastrandrea, ${ }^{148}$

A. Mastroberardino, ${ }^{37 \mathrm{a}, 37 \mathrm{~b}}$ T. Masubuchi, ${ }^{155} \mathrm{P}$. Matricon, ${ }^{115} \mathrm{H}$. Matsunaga, ${ }^{155} \mathrm{~T}$. Matsushita, ${ }^{66} \mathrm{C}$. Mattravers, ${ }^{118, \mathrm{~d}}$ J. Maurer, ${ }^{83}$ S. J. Maxfield, ${ }^{73}$ A. Mayne, ${ }^{139}$ R. Mazini,${ }^{151}$ M. Mazur ${ }^{21}$ L. Mazzaferro, ${ }^{133 a, 133 b}$ M. Mazzanti, ${ }^{89 a}$ J. Mc Donald, ${ }^{85}$ S. P. Mc Kee, ${ }^{87}$ A. McCarn, ${ }^{165}$ R. L. McCarthy, ${ }^{148}$ T. G. McCarthy, ${ }^{29}$ N. A. McCubbin, ${ }^{129}$

K. W. McFarlane, ${ }^{56, a}$ J. A. Mcfayden, ${ }^{139}$ G. Mchedlidze, ${ }^{51 b}$ T. Mclaughlan, ${ }^{18}$ S. J. McMahon, ${ }^{129}$ R. A. McPherson, ${ }^{169,1}$ A. Meade, ${ }^{84}$ J. Mechnich, ${ }^{105}$ M. Mechtel, ${ }^{175}$ M. Medinnis, ${ }^{42}$ R. Meera-Lebbai,,${ }^{11}$ T. Meguro, ${ }^{116}$ R. Mehdiyev,${ }^{93}$ S. Mehlhase, ${ }^{36}$ A. Mehta, ${ }^{73}$ K. Meier,${ }^{58 a}$ B. Meirose,${ }^{79}$ C. Melachrinos,${ }^{31}$ 
B. R. Mellado Garcia, ${ }^{173}$ F. Meloni, ${ }^{89 a, 89 b}$ L. Mendoza Navas, ${ }^{162}$ Z. Meng, ${ }^{151, v}$ A. Mengarelli, ${ }^{20 a, 20 b}$ S. Menke, ${ }^{99}$

E. Meoni, ${ }^{161}$ K. M. Mercurio ${ }^{57}$ P. Mermod, ${ }^{49}$ L. Merola,${ }^{102 a, 102 b}$ C. Meroni, ${ }^{89 a}$ F. S. Merritt,${ }^{31}$ H. Merritt, ${ }^{109}$

A. Messina, ${ }^{30, z}$ J. Metcalfe, ${ }^{25}$ A. S. Mete, ${ }^{163}$ C. Meyer, ${ }^{81}$ C. Meyer, ${ }^{31}$ J-P. Meyer, ${ }^{136}$ J. Meyer, ${ }^{174}$ J. Meyer, ${ }^{54}$

T. C. Meyer ${ }^{30}$ J. Miao, ${ }^{33 d}$ S. Michal,${ }^{30}$ L. Micu, ${ }^{26 a}$ R. P. Middleton, ${ }^{129}$ S. Migas, ${ }^{73}$ L. Mijović, ${ }^{136}$ G. Mikenberg, ${ }^{172}$ M. Mikestikova, ${ }^{125}$ M. Mikuž,${ }^{74}$ D. W. Miller, ${ }^{31}$ R. J. Miller, ${ }^{88}$ W. J. Mills, ${ }^{168}$ C. Mills, ${ }^{57}$ A. Milov, ${ }^{172}$

D. A. Milstead, ${ }^{146 a, 146 b}$ D. Milstein, ${ }^{172}$ A. A. Minaenko, ${ }^{128}$ M. Miñano Moya, ${ }^{167}$ I. A. Minashvili, ${ }^{64}$ A. I. Mincer, ${ }^{108}$ B. Mindur, ${ }^{38}$ M. Mineev, ${ }^{64}$ Y. Ming, ${ }^{173}$ L. M. Mir, ${ }^{12}$ G. Mirabelli, ${ }^{132 a}$ J. Mitrevski, ${ }^{137}$ V. A. Mitsou, ${ }^{167}$ S. Mitsui ${ }^{65}$ P. S. Miyagawa, ${ }^{139}$ J. U. Mjörnmark, ${ }^{79}$ T. Moa,${ }^{146 a, 146 b}$ V. Moeller, ${ }^{28}$ K. Mönig, ${ }^{42}$ N. Möser,${ }^{21}$ S. Mohapatra, ${ }^{148}$

W. Mohr,${ }^{48}$ R. Moles-Valls ${ }^{167}$ J. Monk,${ }^{77}$ E. Monnier, ${ }^{83}$ J. Montejo Berlingen, ${ }^{12}$ F. Monticelli, ${ }^{70}$ S. Monzani,${ }^{20 a, 20 b}$ R. W. Moore, ${ }^{3}$ G. F. Moorhead, ${ }^{86}$ C. Mora Herrera, ${ }^{49}$ A. Moraes, ${ }^{53}$ N. Morange, ${ }^{136}$ J. Morel,${ }^{54}$ G. Morello, ${ }^{37 a, 37 b}$ D. Moreno, ${ }^{81}$ M. Moreno Llácer, ${ }^{167}$ P. Morettini, ${ }^{50 a}$ M. Morgenstern, ${ }^{44}$ M. Morii, ${ }^{57}$ A. K. Morley, ${ }^{30}$ G. Mornacchi, ${ }^{30}$

J. D. Morris, ${ }^{75}$ L. Morvaj, ${ }^{101}$ H. G. Moser, ${ }^{99}$ M. Mosidze, ${ }^{51 b}$ J. Moss, ${ }^{109}$ R. Mount,${ }^{143}$ E. Mountricha, ${ }^{10, a a}$ S. V. Mouraviev, ${ }^{94, a}$ E. J. W. Moyse ${ }^{84}$ F. Mueller, ${ }^{58 a}$ J. Mueller, ${ }^{123}$ K. Mueller, ${ }^{21}$ T. A. Müller, ${ }^{98}$ T. Mueller, ${ }^{81}$ D. Muenstermann, ${ }^{30}$ Y. Munwes, ${ }^{153}$ W. J. Murray,${ }^{129}$ I. Mussche, ${ }^{105}$ E. Musto, ${ }^{102 a, 102 b}$ A. G. Myagkov, ${ }^{128}$ M. Myska, ${ }^{125}$ J. Nadal, ${ }^{12}$ K. Nagai, ${ }^{160}$ R. Nagai, ${ }^{157}$ K. Nagano, ${ }^{65}$ A. Nagarkar, ${ }^{109}$ Y. Nagasaka, ${ }^{59}$ M. Nagel,${ }^{99}$ A. M. Nairz,${ }^{30}$ Y. Nakahama ${ }^{30}$ K. Nakamura,${ }^{155}$ T. Nakamura, ${ }^{155}$ I. Nakano, ${ }^{110}$ G. Nanava, ${ }^{21}$ A. Napier, ${ }^{161}$ R. Narayan, ${ }^{58 \mathrm{~b}}$ M. Nash, ${ }^{77, \mathrm{~d}}$ T. Nattermann, ${ }^{21}$ T. Naumann, ${ }^{42}$ G. Navarro, ${ }^{162}$ H. A. Neal, ${ }^{87}$ P. Yu. Nechaeva, ${ }^{94}$ T. J. Neep, ${ }^{82}$ A. Negri, ${ }^{119 a, 119 b}$ G. Negri, ${ }^{30}$ M. Negrini, ${ }^{20 a}$ S. Nektarijevic, ${ }^{49}$ A. Nelson, ${ }^{163}$ T. K. Nelson, ${ }^{143}$ S. Nemecek, ${ }^{125}$ P. Nemethy, ${ }^{108}$ A. A. Nepomuceno, ${ }^{24 a}$ M. Nessi, ${ }^{30, b b}$ M. S. Neubauer, ${ }^{165}$ M. Neumann,,${ }^{175}$ A. Neusiedl ${ }^{81}$ R. M. Neves, ${ }^{108}$ P. Nevski, ${ }^{25}$ P. R. Newman, ${ }^{18}$ V. Nguyen Thi Hong, ${ }^{136}$ R. B. Nickerson,,${ }^{118}$ R. Nicolaidou, ${ }^{136}$ B. Nicquevert,${ }^{30}$ F. Niedercorn, ${ }^{115}$ J. Nielsen, ${ }^{137}$ N. Nikiforou, ${ }^{35}$ A. Nikiforov, ${ }^{16}$ V. Nikolaenko, ${ }^{128}$ I. Nikolic-Audit, ${ }^{78}$ K. Nikolics, ${ }^{49}$ K. Nikolopoulos, ${ }^{18}$ H. Nilsen, ${ }^{48}$ P. Nilsson, ${ }^{8}$ Y. Ninomiya,${ }^{155}$ A. Nisati, ${ }^{132 a}$ R. Nisius, ${ }^{99}$ T. Nobe ${ }^{157}$ L. Nodulman, ${ }^{6}$ M. Nomachi, ${ }^{116}$ I. Nomidis, ${ }^{154}$ S. Norberg, ${ }^{111}$ M. Nordberg, ${ }^{30}$ P. R. Norton, ${ }^{129}$ J. Novakova, ${ }^{126}$ M. Nozaki, ${ }^{65}$ L. Nozka, ${ }^{113}$ I. M. Nugent, ${ }^{159 a}$ A.-E. Nuncio-Quiroz, ${ }^{21}$ G. Nunes Hanninger, ${ }^{86}$ T. Nunnemann, ${ }^{98}$ E. Nurse, ${ }^{77}$ B. J. O’Brien, ${ }^{46}$ D. C. O’Neil, ${ }^{142}$ V. O'Shea, ${ }^{53}$ L. B. Oakes, ${ }^{98}$ F. G. Oakham, ${ }^{29, e}$ H. Oberlack, ${ }^{99}$ J. Ocariz ${ }^{78}$ A. Ochi, ${ }^{66}$ S. Oda, ${ }^{69}$ S. Odaka, ${ }^{65}$ J. Odier, ${ }^{83}$ H. Ogren, ${ }^{60}$ A. Oh, ${ }^{82}$

S. H. Oh, ${ }^{45}$ C. C. Ohm,${ }^{30}$ T. Ohshima,${ }^{101}$ H. Okawa, ${ }^{25}$ Y. Okumura, ${ }^{31}$ T. Okuyama, ${ }^{155}$ A. Olariu, ${ }^{26 a}$

A. G. Olchevski, ${ }^{64}$ S. A. Olivares Pino, ${ }^{32 a}$ M. Oliveira, ${ }^{124 a, i}$ D. Oliveira Damazio, ${ }^{25}$ E. Oliver Garcia, ${ }^{167}$ D. Olivito, ${ }^{120}$ A. Olszewski, ${ }^{39}$ J. Olszowska, ${ }^{39}$ A. Onofre, ${ }^{124 a, c c}$ P. U. E. Onyisi, ${ }^{31}$ C. J. Oram, ${ }^{159 a}$ M. J. Oreglia, ${ }^{31}$ Y. Oren, ${ }^{153}$

D. Orestano, ${ }^{134 a, 134 b}$ N. Orlando, ${ }^{72 a, 72 b}$ I. Orlov, ${ }^{107}$ C. Oropeza Barrera, ${ }^{53}$ R. S. Orr, ${ }^{158}$ B. Osculati, ${ }^{50 a, 50 b}$ R. Ospanov, ${ }^{120}$ C. Osuna ${ }^{12}$ G. Otero y Garzon, ${ }^{27}$ J.P. Ottersbach, ${ }^{105}$ M. Ouchrif, ${ }^{135 \mathrm{~d}}$ E. A. Ouellette, ${ }^{169}$ F. Ould-Saada, ${ }^{117}$ A. Ouraou, ${ }^{136}$ Q. Ouyang, ${ }^{33 a}$ A. Ovcharova, ${ }^{15}$ M. Owen, ${ }^{82}$ S. Owen, ${ }^{139}$ V. E. Ozcan, ${ }^{19 a}$ N. Ozturk, ${ }^{8}$ A. Pacheco Pages,${ }^{12}$ C. Padilla Aranda,${ }^{12}$ S. Pagan Griso, ${ }^{15}$ E. Paganis,${ }^{139}$ C. Pahl,${ }^{99}$ F. Paige,${ }^{25}$ P. Pais,${ }^{84}$ K. Pajchel, ${ }^{117}$ G. Palacino, ${ }^{159 b}$ C. P. Paleari, ${ }^{7}$ S. Palestini, ${ }^{30}$ D. Pallin, ${ }^{34}$ A. Palma, ${ }^{124 a}$ J. D. Palmer, ${ }^{18}$ Y. B. Pan, ${ }^{173}$ E. Panagiotopoulou, ${ }^{10}$ P. Pani, ${ }^{105}$ N. Panikashvili, ${ }^{87}$ S. Panitkin, ${ }^{25}$ D. Pantea, ${ }^{26 a}$ A. Papadelis, ${ }^{146 a}$

Th. D. Papadopoulou ${ }^{10}$ A. Paramonov, ${ }^{6}$ D. Paredes Hernandez,${ }^{34}$ W. Park, ${ }^{25, d d}$ M. A. Parker,${ }^{28}$ F. Parodi,,${ }^{50,50 b}$ J. A. Parsons, ${ }^{35}$ U. Parzefall, ${ }^{48}$ S. Pashapour,${ }^{54}$ E. Pasqualucci, ${ }^{132 a}$ S. Passaggio, ${ }^{50 a}$ A. Passeri, ${ }^{134 a}$

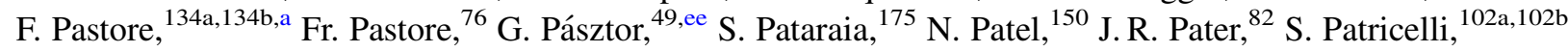
T. Pauly, ${ }^{30}$ M. Pecsy, ${ }^{144 a}$ S. Pedraza Lopez, ${ }^{167}$ M. I. Pedraza Morales, ${ }^{173}$ S. V. Peleganchuk, ${ }^{107}$ D. Pelikan, ${ }^{166}$ H. Peng, ${ }^{33 \mathrm{~b}}$ B. Penning, ${ }^{31}$ A. Penson, ${ }^{35}$ J. Penwell, ${ }^{60}$ M. Perantoni, ${ }^{24 \mathrm{a}}$ K. Perez, ${ }^{35, f f}$ T. Perez Cavalcanti, ${ }^{42}$ E. Perez Codina, ${ }^{159 a}$ M. T. Pérez García-Estañ ${ }^{167}$ V. Perez Reale, ${ }^{35}$ L. Perini, ${ }^{89 a, 89 b}$ H. Pernegger, ${ }^{30}$ R. Perrino, ${ }^{72 a}$ P. Perrodo, ${ }^{5}$ V. D. Peshekhonov, ${ }^{64}$ K. Peters,${ }^{30}$ B. A. Petersen,${ }^{30}$ J. Petersen, ${ }^{30}$ T. C. Petersen,${ }^{36}$ E. Petit, ${ }^{5}$ A. Petridis, ${ }^{154}$ C. Petridou, ${ }^{154}$ E. Petrolo, ${ }^{132 a}$ F. Petrucci, ${ }^{134 a, 134 b}$ D. Petschull, ${ }^{42}$ M. Petteni, ${ }^{142}$ R. Pezoa, ${ }^{32 b}$ A. Phan ${ }^{86}$ P. W. Phillips,${ }^{129}$ G. Piacquadio, ${ }^{30}$ A. Picazio, ${ }^{49}$ E. Piccaro, ${ }^{75}$ M. Piccinini, ${ }^{20 a, 20 b}$ S. M. Piec, ${ }^{42}$ R. Piegaia, ${ }^{27}$ D. T. Pignotti, ${ }^{109}$ J. E. Pilcher, ${ }^{31}$ A. D. Pilkington, ${ }^{82}$ J. Pina, ${ }^{124 a, c}$ M. Pinamonti, ${ }^{164 a, 164 c}$ A. Pinder, ${ }^{118}$ J. L. Pinfold, ${ }^{3}$ B. Pinto, ${ }^{124 a}$ C. Pizio, ${ }^{89 a, 89 b}$ M. Plamondon, ${ }^{169}$ M.-A. Pleier, ${ }^{25}$ E. Plotnikova, ${ }^{64}$ A. Poblaguev, ${ }^{25}$ S. Poddar ${ }^{58 a}$ F. Podlyski, ${ }^{34}$ L. Poggioli, ${ }^{115}$ D. Pohl,${ }^{21}$ M. Pohl, ${ }^{49}$ G. Polesello, ${ }^{119 a}$ A. Policicchio, ${ }^{37 a, 37 b}$ A. Polini, ${ }^{20 a}$ J. Poll ${ }^{75}$ V. Polychronakos, ${ }^{25}$ D. Pomeroy, ${ }^{23}$ K. Pommès, ${ }^{30}$ L. Pontecorvo, ${ }^{132 a}$ B. G. Pope,${ }^{88}$ G. A. Popeneciu, ${ }^{26 a}$ D. S. Popovic, ${ }^{13 a}$ A. Poppleton, ${ }^{30}$ X. Portell Bueso, ${ }^{30}$ G. E. Pospelov, ${ }^{99}$ S. Pospisil, ${ }^{127}$ I. N. Potrap,${ }^{99}$ C. J. Potter, ${ }^{149}$ C. T. Potter, ${ }^{114}$ G. Poulard ${ }^{30}$ J. Poveda, ${ }^{60}$ V. Pozdnyakov, ${ }^{64}$ R. Prabhu,${ }^{77}$ P. Pralavorio, ${ }^{83}$ A. Pranko, ${ }^{15}$ S. Prasad, ${ }^{30}$ R. Pravahan, ${ }^{25}$ S. Prell,${ }^{63}$ K. Pretzl,${ }^{17}$ D. Price,${ }^{60}$ J. Price,${ }^{73}$ L. E. Price,${ }^{6}$ D. Prieur, ${ }^{123}$ M. Primavera, ${ }^{72 a}$ 
K. Prokofiev, ${ }^{108}$ F. Prokoshin, ${ }^{32 b}$ S. Protopopescu, ${ }^{25}$ J. Proudfoot, ${ }^{6}$ X. Prudent, ${ }^{44}$ M. Przybycien, ${ }^{38}$ H. Przysiezniak, ${ }^{5}$ S. Psoroulas, ${ }^{21}$ E. Ptacek, ${ }^{114}$ E. Pueschel,${ }^{84}$ J. Purdham,${ }^{87}$ M. Purohit ${ }^{25, \text { dd }}$ P. Puzo, ${ }^{115}$ Y. Pylypchenko, ${ }^{62}$ J. Qian,${ }^{87}$ A. Quadt, ${ }^{54}$ D. R. Quarrie, ${ }^{15}$ W. B. Quayle, ${ }^{173}$ F. Quinonez, ${ }^{32 a}$ M. Raas, ${ }^{104}$ V. Radescu, ${ }^{42}$ P. Radloff, ${ }^{114}$ T. Rador, ${ }^{19 a}$ F. Ragusa, ${ }^{89 a, 89 b}$ G. Rahal, ${ }^{178}$ A. M. Rahimi, ${ }^{109}$ D. Rahm, ${ }^{25}$ S. Rajagopalan, ${ }^{25}$ M. Rammensee,${ }^{48}$ M. Rammes, ${ }^{141}$ A. S. Randle-Conde ${ }^{40}$ K. Randrianarivony, ${ }^{29}$ F. Rauscher, ${ }^{98}$ T. C. Rave, ${ }^{48}$ M. Raymond, ${ }^{30}$ A. L. Read, ${ }^{117}$ D. M. Rebuzzi, ${ }^{19 a, 119 b}$ A. Redelbach,,${ }^{174}$ G. Redlinger, ${ }^{25}$ R. Reece, ${ }^{120}$ K. Reeves, ${ }^{41}$ E. Reinherz-Aronis, ${ }^{153}$ A. Reinsch, ${ }^{114}$ I. Reisinger, ${ }^{43}$ C. Rembser ${ }^{30}$ Z. L. Ren, ${ }^{151}$ A. Renaud, ${ }^{115}$ M. Rescigno, ${ }^{132 a}$ S. Resconi, ${ }^{89 a}$ B. Resende, ${ }^{136}$ P. Reznicek, ${ }^{98}$ R. Rezvani, ${ }^{158}$ R. Richter, ${ }^{99}$ E. Richter-Was, ${ }^{5, g g}$ M. Ridel,${ }^{78}$ M. Rijpstra, ${ }^{105}$ M. Rijssenbeek, ${ }^{148}$ A. Rimoldi, ${ }^{119 a, 119 b}$ L. Rinaldi, ${ }^{20 a}$ R. R. Rios,${ }^{40}$ I. Riu, ${ }^{12}$ G. Rivoltella, ${ }^{89 a, 89 b}$ F. Rizatdinova, ${ }^{112}$ E. Rizvi, ${ }^{75}$ S. H. Robertson, ${ }^{85,1}$ A. Robichaud-Veronneau, ${ }^{118}$ D. Robinson, ${ }^{28}$ J. E. M. Robinson, ${ }^{82}$ A. Robson, ${ }^{53}$ J. G. Rocha de Lima, ${ }^{106}$ C. Roda, ${ }^{122 a, 122 b}$ D. Roda Dos Santos,${ }^{30}$ A. Roe,${ }^{54}$ S. Roe,${ }^{30}$ O. Røhne, ${ }^{117}$ S. Rolli, ${ }^{161}$ A. Romaniouk, ${ }^{96}$ M. Romano, ${ }^{20 a, 20 b}$ G. Romeo, ${ }^{27}$ E. Romero Adam, ${ }^{167}$ N. Rompotis, ${ }^{138}$ L. Roos, ${ }^{78}$ E. Ros, ${ }^{167}$ S. Rosati, ${ }^{132 a}$ K. Rosbach, ${ }^{49}$ A. Rose,${ }^{149}$ M. Rose, ${ }^{76}$ G. A. Rosenbaum, ${ }^{158}$ E. I. Rosenberg, ${ }^{63}$ P. L. Rosendahl, ${ }^{14}$ O. Rosenthal, ${ }^{141}$ L. Rosselet, ${ }^{49}$ V. Rossetti, ${ }^{12}$ E. Rossi, ${ }^{132 a \mathrm{a}, 132 \mathrm{~b}}$ L. P. Rossi, ${ }^{50 \mathrm{a}}$ M. Rotaru, ${ }^{26 \mathrm{a}}$ I. Roth, ${ }^{172}$ J. Rothberg, ${ }^{138}$ D. Rousseau, ${ }^{115}$ C. R. Royon, ${ }^{136}$ A. Rozanov, ${ }^{83}$ Y. Rozen, ${ }^{152}$ X. Ruan, ${ }^{33 a, h h}$ F. Rubbo, ${ }^{12}$ I. Rubinskiy, ${ }^{42}$ N. Ruckstuhl, ${ }^{105}$ V. I. Rud, ${ }^{97}$ C. Rudolph,${ }^{44}$ G. Rudolph, ${ }^{61}$ F. Rühr, ${ }^{7}$ A. Ruiz-Martinez, ${ }^{63}$ L. Rumyantsev, ${ }^{64}$ Z. Rurikova, ${ }^{48}$ N. A. Rusakovich, ${ }^{64}$ J. P. Rutherfoord, ${ }^{7}$ C. Ruwiedel, ${ }^{15, a}$ P. Ruzicka, ${ }^{125}$ Y. F. Ryabov, ${ }^{121}$ M. Rybar, ${ }^{126}$ G. Rybkin, ${ }^{115}$ N. C. Ryder, ${ }^{18}$ A. F. Saavedra, ${ }^{150}$ I. Sadeh, ${ }^{153}$ H. F-W. Sadrozinski, ${ }^{137}$ R. Sadykov, ${ }^{64}$

F. Safai Tehrani, ${ }^{132 a}$ H. Sakamoto, ${ }^{155}$ G. Salamanna ${ }^{75}$ A. Salamon, ${ }^{133 a}$ M. Saleem, ${ }^{111}$ D. Salek, ${ }^{30}$ D. Salihagic,${ }^{99}$ A. Salnikov, ${ }^{143}$ J. Salt, ${ }^{167}$ B. M. Salvachua Ferrando, ${ }^{6}$ D. Salvatore,${ }^{37 a, 37 b}$ F. Salvatore, ${ }^{149}$ A. Salvucci, ${ }^{104}$ A. Salzburger, ${ }^{30}$ D. Sampsonidis, ${ }^{154}$ B. H. Samset, ${ }^{117}$ A. Sanchez, ${ }^{102 a, 102 b}$ V. Sanchez Martinez, ${ }^{167}$ H. Sandaker, ${ }^{14}$ H. G. Sander, ${ }^{81}$ M. P. Sanders, ${ }^{98}$ M. Sandhoff, ${ }^{175}$ T. Sandoval, ${ }^{28}$ C. Sandoval, ${ }^{162}$ R. Sandstroem, ${ }^{99}$ D. P. C. Sankey, ${ }^{129}$ A. Sansoni, ${ }^{47}$ C. Santamarina Rios,${ }^{85}$ C. Santoni,${ }^{34}$ R. Santonico, ${ }^{133 a, 133 b}$ H. Santos, ${ }^{124 a}$ J. G. Saraiva, ${ }^{124 a}$ T. Sarangi, ${ }^{173}$ E. Sarkisyan-Grinbaum, ${ }^{8}$ F. Sarri, ${ }^{122 a, 122 b}$ G. Sartisohn, ${ }^{175}$ O. Sasaki, ${ }^{65}$ Y. Sasaki, ${ }^{155}$ N. Sasao, ${ }^{67}$ I. Satsounkevitch, ${ }^{90}$ G. Sauvage, ${ }^{5, a}$ E. Sauvan, ${ }^{5}$ J. B. Sauvan, ${ }^{115}$ P. Savard, ${ }^{158, e}$ V. Savinov, ${ }^{123}$ D. O. Savu, ${ }^{30}$ L. Sawyer, ${ }^{25, n}$ D. H. Saxon, ${ }^{53}$ J. Saxon, ${ }^{120}$ C. Sbarra, ${ }^{20 a}$ A. Sbrizzi, ${ }^{20 a, 20 b}$ D. A. Scannicchio, ${ }^{163}$ M. Scarcella, ${ }^{150}$ J. Schaarschmidt, ${ }^{15}$ P. Schacht,,${ }^{99}$ D. Schaefer, ${ }^{120}$ U. Schäfer ${ }^{81}$ S. Schaepe, ${ }^{21}$ S. Schaetzel, ${ }^{58 b}$ A. C. Schaffer, ${ }^{115}$ D. Schaile, ${ }^{98}$ R. D. Schamberger, ${ }^{148}$ A. G. Schamov, ${ }^{107}$ V. Scharf, ${ }^{58 a}$ V. A. Schegelsky, ${ }^{121}$ D. Scheirich, ${ }^{87}$ M. Schernau, ${ }^{163}$ M. I. Scherzer, ${ }^{35}$ C. Schiavi, ${ }^{50 a, 50 b}$ J. Schieck, ${ }^{98}$ M. Schioppa,${ }^{37 a, 37 b}$ S. Schlenker, ${ }^{30}$ E. Schmidt, ${ }^{48}$ K. Schmieden, ${ }^{21}$ C. Schmitt, ${ }^{81}$ S. Schmitt, ${ }^{58 b}$ M. Schmitz, ${ }^{21}$ B. Schneider, ${ }^{17}$ U. Schnoor, ${ }^{44}$ A. Schoening, ${ }^{58 b}$

A. L. S. Schorlemmer, ${ }^{54}$ M. Schott,${ }^{30}$ D. Schouten, ${ }^{159 a}$ J. Schovancova, ${ }^{125}$ M. Schram, ${ }^{85}$ C. Schroeder, ${ }^{81}$ N. Schroer, ${ }^{58 c}$ M. J. Schultens, ${ }^{21}$ J. Schultes, ${ }^{175}$ H.-C. Schultz-Coulon, ${ }^{58 a}$ H. Schulz, ${ }^{16}$ M. Schumacher, ${ }^{48}$ B. A. Schumm, ${ }^{137} \mathrm{Ph}$. Schune, ${ }^{136}$ C. Schwanenberger, ${ }^{82}$ A. Schwartzman, ${ }^{143} \mathrm{Ph}$. Schwegler, ${ }^{99} \mathrm{Ph}$. Schwemling ${ }^{78}$ R. Schwienhorst, ${ }^{88}$ R. Schwierz, ${ }^{44}$ J. Schwindling, ${ }^{136}$ T. Schwindt, ${ }^{21}$ M. Schwoerer, ${ }^{5}$ G. Sciolla, ${ }^{23}$ W. G. Scott, ${ }^{129}$ J. Searcy, ${ }^{114}$ G. Sedov, ${ }^{42}$ E. Sedykh, ${ }^{121}$ S. C. Seidel, ${ }^{103}$ A. Seiden, ${ }^{137}$ F. Seifert, ${ }^{44}$ J. M. Seixas, ${ }^{24 a}$ G. Sekhniaidze, ${ }^{102 a}$ S. J. Sekula, ${ }^{40}$ K. E. Selbach, ${ }^{46}$ D. M. Seliverstov, ${ }^{121}$ B. Sellden, ${ }^{146 a}$ G. Sellers, ${ }^{73}$ M. Seman,${ }^{144 b}$

N. Semprini-Cesari, ${ }^{20 a, 20 b}$ C. Serfon, ${ }^{98}$ L. Serin,,${ }^{15}$ L. Serkin, ${ }^{54}$ R. Seuster, ${ }^{99}$ H. Severini, ${ }^{111}$ A. Sfyrla, ${ }^{30}$ E. Shabalina, ${ }^{54}$ M. Shamim, ${ }^{114}$ L. Y. Shan, ${ }^{33 a}$ J. T. Shank, ${ }^{22}$ Q. T. Shao,${ }^{86}$ M. Shapiro, ${ }^{15}$ P. B. Shatalov, ${ }^{95}$ K. Shaw, ${ }^{164 a, 164 c}$ D. Sherman, ${ }^{176}$ P. Sherwood, ${ }^{77}$ A. Shibata, ${ }^{108}$ S. Shimizu, ${ }^{101}$ M. Shimojima, ${ }^{100}$ T. Shin, ${ }^{56}$ M. Shiyakova, ${ }^{64}$ A. Shmeleva, ${ }^{94}$ M. J. Shochet, ${ }^{31}$ D. Short, ${ }^{118}$ S. Shrestha,${ }^{63}$ E. Shulga,${ }^{96}$ M. A. Shupe, ${ }^{7}$ P. Sicho, ${ }^{125}$ A. Sidoti, ${ }^{132 a}$ F. Siegert, ${ }^{48}$ Dj. Sijacki, ${ }^{13 a}$ O. Silbert, ${ }^{172}$ J. Silva, ${ }^{124 a}$ Y. Silver ${ }^{153}$ D. Silverstein, ${ }^{143}$

S. B. Silverstein, ${ }^{146 a}$ V. Simak, ${ }^{127}$ O. Simard,${ }^{136}$ Lj. Simic, ${ }^{13 a}$ S. Simion, ${ }^{115}$ E. Simioni,${ }^{81}$ B. Simmons,${ }^{77}$ R. Simoniello, ${ }^{89 a, 89 b}$ M. Simonyan, ${ }^{36}$ P. Sinervo, ${ }^{158}$ N. B. Sinev, ${ }^{114}$ V. Sipica, ${ }^{141}$ G. Siragusa, ${ }^{174}$ A. Sircar, ${ }^{25}$ A. N. Sisakyan, ${ }^{64, a}$ S. Yu. Sivoklokov, ${ }^{97}$ J. Sjölin, ${ }^{146 a, 146 b}$ T. B. Sjursen, ${ }^{14}$ L. A. Skinnari, ${ }^{15}$ H. P. Skottowe,${ }^{57}$ K. Skovpen, ${ }^{107}$ P. Skubic, ${ }^{111}$ M. Slater, ${ }^{18}$ T. Slavicek, ${ }^{127}$ K. Sliwa, ${ }^{161}$ V. Smakhtin, ${ }^{172}$ B. H. Smart, ${ }^{46}$ S. L. Smestad, ${ }^{117}$ S. Yu. Smirnov, ${ }^{96}$ Y. Smirnov, ${ }^{96}$ L. N. Smirnova, ${ }^{97}$ O. Smirnova, ${ }^{79}$ B. C. Smith, ${ }^{57}$ D. Smith, ${ }^{143}$ K. M. Smith, ${ }^{53}$ M. Smizanska, ${ }^{71}$ K. Smolek, ${ }^{127}$ A. A. Snesarev,${ }^{94}$ S. W. Snow, ${ }^{82}$ J. Snow, ${ }^{111}$ S. Snyder, ${ }^{25}$ R. Sobie, ${ }^{169,1}$ J. Sodomka, ${ }^{127}$ A. Soffer, ${ }^{153}$ C. A. Solans, ${ }^{167}$ M. Solar, ${ }^{127}$ J. Solc, ${ }^{127}$ E. Yu. Soldatov, ${ }^{96}$ U. Soldevila, ${ }^{167}$

E. Solfaroli Camillocci, ${ }^{132 a, 132 b}$ A. A. Solodkov, ${ }^{128}$ O. V. Solovyanov, ${ }^{128}$ V. Solovyev, ${ }^{121}$ N. Soni, ${ }^{1}$ V. Sopko, ${ }^{127}$ B. Sopko, ${ }^{127}$ M. Sosebee, ${ }^{8}$ R. Soualah, ${ }^{164 a, 164 c}$ A. Soukharev, ${ }^{107}$ S. Spagnolo, ${ }^{72 a, 72 b}$ F. Spanò,${ }^{76}$ R. Spighi, ${ }^{20 a}$ G. Spigo, ${ }^{30}$ R. Spiwoks, ${ }^{30}$ M. Spousta, ${ }^{126, i i}$ T. Spreitzer,${ }^{158}$ B. Spurlock, ${ }^{8}$ R. D. St. Denis,${ }^{53}$ J. Stahlman, ${ }^{120}$ 
R. Stamen, ${ }^{58 a}$ E. Stanecka, ${ }^{39}$ R. W. Stanek, ${ }^{6}$ C. Stanescu, ${ }^{134 a}$ M. Stanescu-Bellu, ${ }^{42}$ M. M. Stanitzki, ${ }^{42}$ S. Stapnes, ${ }^{117}$ E. A. Starchenko, ${ }^{128}$ J. Stark, ${ }^{55}$ P. Staroba ${ }^{125}$ P. Starovoitov, ${ }^{42}$ R. Staszewski, ${ }^{39}$ A. Staude, ${ }^{98}$ P. Stavina, ${ }^{144 a, a}$ G. Steele, ${ }^{53}$ P. Steinbach, ${ }^{44}$ P. Steinberg, ${ }^{25}$ I. Stekl,${ }^{127}$ B. Stelzer, ${ }^{142}$ H. J. Stelzer,${ }^{88}$ O. Stelzer-Chilton, ${ }^{159 a}$ H. Stenzel, ${ }^{52}$ S. Stern, ${ }^{99}$ G. A. Stewart, ${ }^{30}$ J. A. Stillings, ${ }^{21}$ M. C. Stockton, ${ }^{85}$ K. Stoerig, ${ }^{48}$ G. Stoicea, ${ }^{26 a}$ S. Stonjek, ${ }^{99}$ P. Strachota, ${ }^{126}$ A. R. Stradling, ${ }^{8}$ A. Straessner, ${ }^{44}$ J. Strandberg, ${ }^{147}$ S. Strandberg, ${ }^{146 a, 146 b}$ A. Strandlie, ${ }^{117}$ M. Strang, ${ }^{109}$ E. Strauss, ${ }^{143}$ M. Strauss, ${ }^{111}$ P. Strizenec, ${ }^{144 b}$ R. Ströhmer, ${ }^{174}$ D. M. Strom, ${ }^{114}$ J. A. Strong, ${ }^{76, a}$ R. Stroynowski, ${ }^{40}$ J. Strube, ${ }^{129}$ B. Stugu, ${ }^{14}$ I. Stumer, ${ }^{25, a}$ J. Stupak, ${ }^{148}$ P. Sturm, ${ }^{175}$ N. A. Styles, ${ }^{42}$ D. A. Soh, ${ }^{151, x}$ D. Su, ${ }^{143}$ HS. Subramania, ${ }^{3}$ A. Succurro, ${ }^{12}$ Y. Sugaya,${ }^{116}$ C. Suhr, ${ }^{106}$ M. Suk, ${ }^{126}$ V. V. Sulin, ${ }^{94}$ S. Sultansoy, ${ }^{4 d}$ T. Sumida,${ }^{67}$ X. Sun,${ }^{55}$ J. E. Sundermann, ${ }^{48}$ K. Suruliz, ${ }^{139}$ G. Susinno,,${ }^{37 a, 37 b}$ M. R. Sutton, ${ }^{149}$ Y. Suzuki, ${ }^{65}$ Y. Suzuki, ${ }^{66}$ M. Svatos, ${ }^{125}$ S. Swedish, ${ }^{168}$ I. Sykora, ${ }^{144 a}$ T. Sykora, ${ }^{126}$ J. Sánchez, ${ }^{167}$ D. Ta, ${ }^{105}$ K. Tackmann, ${ }^{42}$ A. Taffard, ${ }^{163}$ R. Tafirout, ${ }^{159 a}$ N. Taiblum, ${ }^{153}$ Y. Takahashi, ${ }^{101}$ H. Takai, ${ }^{25}$ R. Takashima, ${ }^{68}$ H. Takeda, ${ }^{66}$ T. Takeshita ${ }^{140}$ Y. Takubo,${ }^{65}$ M. Talby,${ }^{83}$ A. Talyshev, ${ }^{107, g}$ M. C. Tamsett,${ }^{25}$ J. Tanaka, ${ }^{155}$ R. Tanaka, ${ }^{115}$ S. Tanaka, ${ }^{131}$ S. Tanaka, ${ }^{65}$ A. J. Tanasijczuk, ${ }^{142} \mathrm{~K}$. Tani,${ }^{66} \mathrm{~N}$. Tannoury, ${ }^{83} \mathrm{~S}$. Tapprogge,${ }^{81} \mathrm{D}$. Tardif,${ }^{158} \mathrm{~S}$. Tarem, ${ }^{152} \mathrm{~F}$. Tarrade, ${ }^{29}$ G. F. Tartarelli, ${ }^{89 a}$ P. Tas, ${ }^{126}$ M. Tasevsky, ${ }^{125}$ E. Tassi, ${ }^{37 a, 37 b}$ M. Tatarkhanov, ${ }^{15}$ Y. Tayalati, ${ }^{135 d}$ C. Taylor, ${ }^{77}$ F. E. Taylor, ${ }^{92}$ G. N. Taylor, ${ }^{86}$ W. Taylor, ${ }^{159 b}$ M. Teinturier, ${ }^{115}$ F. A. Teischinger, ${ }^{30}$ M. Teixeira Dias Castanheira, ${ }^{75}$ P. Teixeira-Dias, ${ }^{76}$ K. K. Temming,${ }^{48}$ H. Ten Kate,${ }^{30}$ P. K. Teng,${ }^{151}$ S. Terada,${ }^{65}$ K. Terashi, ${ }^{155}$ J. Terron,${ }^{80}$ M. Testa, ${ }^{47}$

R. J. Teuscher, ${ }^{158,1}$ J. Therhaag, ${ }^{21}$ T. Theveneaux-Pelzer ${ }^{78}$ S. Thoma, ${ }^{48}$ J. P. Thomas, ${ }^{18}$ E. N. Thompson, ${ }^{35}$

P. D. Thompson, ${ }^{18}$ P. D. Thompson, ${ }^{158}$ A. S. Thompson, ${ }^{53}$ L. A. Thomsen, ${ }^{36}$ E. Thomson, ${ }^{120}$ M. Thomson, ${ }^{28}$ W. M. Thong, ${ }^{86}$ R. P. Thun, ${ }^{87}$ F. Tian,${ }^{35}$ M. J. Tibbetts, ${ }^{15}$ T. Tic,${ }^{125}$ V. O. Tikhomirov, ${ }^{94}$ Y. A. Tikhonov,${ }^{107, g}$ S. Timoshenko, ${ }^{96}$ P. Tipton, ${ }^{176}$ S. Tisserant,${ }^{83}$ T. Todorov, ${ }^{5}$ S. Todorova-Nova, ${ }^{161}$ B. Toggerson, ${ }^{163}$ J. Tojo,${ }^{69}$ S. Tokár, ${ }^{144 a} \mathrm{~K}$. Tokushuku, ${ }^{65} \mathrm{~K}$. Tollefson, ${ }^{88} \mathrm{M}$. Tomoto, ${ }^{101} \mathrm{~L}$. Tompkins, ${ }^{31} \mathrm{~K}$. Toms, ${ }^{103}$ A. Tonoyan, ${ }^{14} \mathrm{C}$. Topfel, ${ }^{17}$

N. D. Topilin, ${ }^{64}$ I. Torchiani, ${ }^{30}$ E. Torrence,${ }^{114}$ H. Torres,${ }^{78}$ E. Torró Pastor, ${ }^{167}$ J. Toth, ${ }^{83, e e}$ F. Touchard, ${ }^{83}$ D. R. Tovey, ${ }^{139}$ T. Trefzger,${ }^{174}$ L. Tremblet, ${ }^{30}$ A. Tricoli, ${ }^{30}$ I. M. Trigger,${ }^{159 a}$ S. Trincaz-Duvoid ${ }^{78}$ M. F. Tripiana, ${ }^{70}$ N. Triplett, ${ }^{25} \mathrm{~W}$. Trischuk, ${ }^{158} \mathrm{~B}$. Trocmé, ${ }^{55} \mathrm{C}$. Troncon, ${ }^{89 \mathrm{a}} \mathrm{M}$. Trottier-McDonald, ${ }^{142} \mathrm{M}$. Trzebinski, ${ }^{39} \mathrm{~A}$. Trzupek, ${ }^{39}$ C. Tsarouchas, ${ }^{30}$ J. C-L. Tseng, ${ }^{118} \mathrm{M}$. Tsiakiris, ${ }^{105} \mathrm{P}$. V. Tsiareshka, ${ }^{90} \mathrm{D}$. Tsionou, ${ }^{5, \mathrm{jj}} \mathrm{G}$. Tsipolitis, ${ }^{10} \mathrm{~S}$. Tsiskaridze, ${ }^{12}$ V. Tsiskaridze, ${ }^{48}$ E. G. Tskhadadze, ${ }^{51 a}$ I. I. Tsukerman, ${ }^{95}$ V. Tsulaia, ${ }^{15}$ J.-W. Tsung, ${ }^{21}$ S. Tsuno, ${ }^{65}$ D. Tsybychev, ${ }^{148}$ A. Tua ${ }^{139}$ A. Tudorache, ${ }^{26 a}$ V. Tudorache, ${ }^{26 a}$ J. M. Tuggle,${ }^{31}$ M. Turala ${ }^{39}$ D. Turecek,${ }^{127}$ I. Turk Cakir, ${ }^{4 e}$ E. Turlay, ${ }^{105}$ R. Turra, ${ }^{89 a, 89 b}$ P. M. Tuts, ${ }^{35}$ A. Tykhonov, ${ }^{74}$ M. Tylmad,,${ }^{146 a, 146 b}$ M. Tyndel, ${ }^{129}$ G. Tzanakos, ${ }^{9}$ K. Uchida, ${ }^{21}$ I. Ueda, ${ }^{155}$ R. Ueno, ${ }^{29}$ M. Ugland, ${ }^{14}$ M. Uhlenbrock,${ }^{21}$ M. Uhrmacher, ${ }^{54}$ F. Ukegawa, ${ }^{160}$ G. Unal, ${ }^{30}$ A. Undrus, ${ }^{25}$ G. Unel, ${ }^{163}$ Y. Unno, ${ }^{65}$ D. Urbaniec,${ }^{35}$ P. Urquijo, ${ }^{21}$ G. Usai, ${ }^{8}$ M. Uslenghi, ${ }^{119 a, 119 b}$ L. Vacavant ${ }^{83}$ V. Vacek, ${ }^{127}$ B. Vachon, ${ }^{85}$ S. Vahsen,${ }^{15}$ J. Valenta, ${ }^{125}$ S. Valentinetti, ${ }^{20 a, 20 b}$ A. Valero, ${ }^{167}$ S. Valkar, ${ }^{126}$

E. Valladolid Gallego, ${ }^{167}$ S. Vallecorsa, ${ }^{152}$ J. A. Valls Ferrer, ${ }^{167}$ P.C. Van Der Deijl, ${ }^{105}$ R. van der Geer, ${ }^{105}$ H. van der Graaf, ${ }^{105}$ R. Van Der Leeuw, ${ }^{105}$ E. van der Poel, ${ }^{105}$ D. van der Ster, ${ }^{30}$ N. van Eldik, ${ }^{30}$ P. van Gemmeren, ${ }^{6}$ I. van Vulpen, ${ }^{105}$ M. Vanadia, ${ }^{99}$ W. Vandelli, ${ }^{30}$ A. Vaniachine, ${ }^{6}$ P. Vankov,${ }^{42}$ F. Vannucci, ${ }^{78}$ R. Vari, ${ }^{132 a}$ T. Varol, ${ }^{84}$

D. Varouchas, ${ }^{15}$ A. Vartapetian, ${ }^{8}$ K. E. Varvell, ${ }^{150}$ V. I. Vassilakopoulos, ${ }^{56}$ F. Vazeille, ${ }^{34}$ T. Vazquez Schroeder, ${ }^{54}$ G. Vegni, ${ }^{89 a, 89 b}$ J. J. Veillet, ${ }^{115}$ F. Veloso, ${ }^{124 a}$ R. Veness, ${ }^{30}$ S. Veneziano, ${ }^{132 a}$ A. Ventura, ${ }^{72 a, 72 b}$ D. Ventura, ${ }^{84}$ M. Venturi, ${ }^{48}$ N. Venturi, ${ }^{158}$ V. Vercesi, ${ }^{119 a}$ M. Verducci, ${ }^{138}$ W. Verkerke, ${ }^{105}$ J. C. Vermeulen, ${ }^{105}$ A. Vest,${ }^{44}$ M. C. Vetterli, ${ }^{142, \mathrm{e}}$ I. Vichou, ${ }^{165}$ T. Vickey, ${ }^{145 b, k k}$ O. E. Vickey Boeriu, ${ }^{145 b}$ G. H. A. Viehhauser, ${ }^{118}$ S. Viel, ${ }^{168}$ M. Villa,${ }^{20 a, 20 b}$ M. Villaplana Perez ${ }^{167}$ E. Vilucchi, ${ }^{47}$ M. G. Vincter, ${ }^{29}$ E. Vinek, ${ }^{30}$ V. B. Vinogradov, ${ }^{64}$ M. Virchaux, ${ }^{136, a}$ J. Virzi,${ }^{15}$ O. Vitells,${ }^{172}$ M. Viti, ${ }^{42}$ I. Vivarelli, ${ }^{48}$ F. Vives Vaque, ${ }^{3}$ S. Vlachos, ${ }^{10}$ D. Vladoiu, ${ }^{98}$ M. Vlasak, ${ }^{127}$ A. Vogel, ${ }^{21}$ P. Vokac, ${ }^{127}$ G. Volpi,${ }^{47}$ M. Volpi,${ }^{86}$ G. Volpini, ${ }^{89 a}$ H. von der Schmitt, ${ }^{99}$ H. von Radziewski ${ }^{48}$ E. von Toerne, ${ }^{21}$ V. Vorobel, ${ }^{126}$ V. Vorwerk, ${ }^{12}$ M. Vos,${ }^{167}$ R. Voss,${ }^{30}$ T. T. Voss,${ }^{175}$ J. H. Vossebeld, ${ }^{73}$ N. Vranjes, ${ }^{136}$ M. Vranjes Milosavljevic, ${ }^{105}$ V. Vrba,${ }^{125}$ M. Vreeswijk, ${ }^{105}$ T. Vu Anh, ${ }^{48}$ R. Vuillermet, ${ }^{30}$ I. Vukotic, ${ }^{31}$ W. Wagner, ${ }^{175}$ P. Wagner, ${ }^{120}$ H. Wahlen, ${ }^{175}$ S. Wahrmund, ${ }^{44}$ J. Wakabayashi, ${ }^{101}$ S. Walch, ${ }^{87}$ J. Walder ${ }^{71}$ R. Walker ${ }^{98}$ W. Walkowiak, ${ }^{141}$ R. Wall, ${ }^{176}$ P. Waller, ${ }^{73}$ B. Walsh, ${ }^{176}$ C. Wang, ${ }^{45}$ H. Wang, ${ }^{173}$

H. Wang, ${ }^{33 b, 11}$ J. Wang, ${ }^{151}$ J. Wang, ${ }^{55}$ R. Wang, ${ }^{103}$ S. M. Wang, ${ }^{151}$ T. Wang, ${ }^{21}$ A. Warburton, ${ }^{85}$ C.P. Ward ${ }^{28}$ M. Warsinsky, ${ }^{48}$ A. Washbrook, ${ }^{46}$ C. Wasicki, ${ }^{42}$ I. Watanabe ${ }^{66}$ P. M. Watkins, ${ }^{18}$ A. T. Watson, ${ }^{18}$ I. J. Watson, ${ }^{150}$ M. F. Watson, ${ }^{18}$ G. Watts, ${ }^{138}$ S. Watts, ${ }^{82}$ A. T. Waugh, ${ }^{150}$ B. M. Waugh,${ }^{77}$ M. S. Weber, ${ }^{17}$ P. Weber, ${ }^{54}$

A. R. Weidberg, ${ }^{118}$ P. Weigell, ${ }^{99}$ J. Weingarten, ${ }^{54}$ C. Weiser, ${ }^{48}$ P. S. Wells, ${ }^{30}$ T. Wenaus, ${ }^{25}$ D. Wendland, ${ }^{16}$ Z. Weng, ${ }^{151, \mathrm{x}} \mathrm{T}$. Wengler, ${ }^{30} \mathrm{~S}$. Wenig, ${ }^{30} \mathrm{~N}$. Wermes, ${ }^{21} \mathrm{M}$. Werner, ${ }^{48} \mathrm{P}$. Werner, ${ }^{30} \mathrm{M}$. Werth, ${ }^{163} \mathrm{M}$. Wessels, ${ }^{58 a}$ J. Wetter, ${ }^{161}$ C. Weydert, ${ }^{55}$ K. Whalen,${ }^{29}$ S. J. Wheeler-Ellis, ${ }^{163}$ A. White, ${ }^{8}$ M. J. White ${ }^{86}$ S. White, ${ }^{122 a, 122 b}$ 
S. R. Whitehead, ${ }^{118}$ D. Whiteson, ${ }^{163}$ D. Whittington, ${ }^{60}$ F. Wicek, ${ }^{115}$ D. Wicke, ${ }^{175}$ F. J. Wickens, ${ }^{129}$ W. Wiedenmann, ${ }^{173}$ M. Wielers, ${ }^{129}$ P. Wienemann, ${ }^{21}$ C. Wiglesworth, ${ }^{75}$ L. A. M. Wiik-Fuchs, ${ }^{48}$ P. A. Wijeratne, ${ }^{77}$ A. Wildauer, ${ }^{99}$ M. A. Wildt, ${ }^{42, t}$ I. Wilhelm, ${ }^{126}$ H. G. Wilkens, ${ }^{30}$ J.Z. Will, ${ }^{98}$ E. Williams,${ }^{35}$ H. H. Williams, ${ }^{120}$ W. Willis ${ }^{35}$ S. Willocq, ${ }^{84}$ J. A. Wilson, ${ }^{18}$ M. G. Wilson, ${ }^{143}$ A. Wilson, ${ }^{87}$ I. Wingerter-Seez, ${ }^{5}$ S. Winkelmann, ${ }^{48}$ F. Winklmeier, ${ }^{30}$ M. Wittgen, ${ }^{143}$ S. J. Wollstadt, ${ }^{81}$ M. W. Wolter, ${ }^{39}$ H. Wolters, ${ }^{124 a, i}$ W. C. Wong, ${ }^{41}$ G. Wooden,${ }^{87}$ B. K. Wosiek, ${ }^{39}$ J. Wotschack, ${ }^{30}$ M. J. Woudstra, ${ }^{82}$ K. W. Wozniak, ${ }^{39}$ K. Wraight, ${ }^{53}$ M. Wright, ${ }^{53}$ B. Wrona, ${ }^{73}$ S. L. Wu,${ }^{173}$ X. Wu ${ }^{49}$ Y. Wu,${ }^{33 b, m m}$ E. Wulf, ${ }^{35}$ B. M. Wynne, ${ }^{46}$ S. Xella, ${ }^{36}$ M. Xiao, ${ }^{136}$ S. Xie, ${ }^{48}$ C. Xu, ${ }^{33 b, a a}$ D. Xu, ${ }^{139}$ B. Yabsley, ${ }^{150}$ S. Yacoob,${ }^{145 a, n n}$ M. Yamada, ${ }^{65}$ H. Yamaguchi, ${ }^{155}$ A. Yamamoto, ${ }^{65}$ K. Yamamoto,${ }^{63}$ S. Yamamoto, ${ }^{155}$ T. Yamamura, ${ }^{155}$ T. Yamanaka, ${ }^{155}$ J. Yamaoka, ${ }^{45}$ T. Yamazaki, ${ }^{155}$ Y. Yamazaki, ${ }^{66}$ Z. Yan, ${ }^{22}$ H. Yang,${ }^{87}$ U. K. Yang,${ }^{82}$ Y. Yang, ${ }^{60}$ Z. Yang, ${ }^{146 a, 146 b}$ S. Yanush, ${ }^{91}$ L. Yao, ${ }^{33 a}$ Y. Yao, ${ }^{15}$ Y. Yasu, ${ }^{65}$ G. V. Ybeles Smit, ${ }^{130}$ J. Ye,${ }^{40}$ S. Ye, ${ }^{25}$ M. Yilmaz, ${ }^{4 \mathrm{c}}$ R. Yoosoofmiya, ${ }^{123}$ K. Yorita, ${ }^{171}$ R. Yoshida, ${ }^{6}$ C. Young, ${ }^{143}$ C. J. Young, ${ }^{118}$ S. Youssef,${ }^{22}$ D. Yu, ${ }^{25}$

J. Yu, ${ }^{8}$ J. Yu, ${ }^{112}$ L. Yuan, ${ }^{66}$ A. Yurkewicz, ${ }^{106}$ M. Byszewski, ${ }^{30}$ B. Zabinski, ${ }^{39}$ R. Zaidan, ${ }^{62}$ A. M. Zaitsev, ${ }^{128}$ Z. Zajacova, ${ }^{30}$ L. Zanello, ${ }^{132 a, 132 b}$ D. Zanzi, ${ }^{99}$ A. Zaytsev, ${ }^{25}$ C. Zeitnitz, ${ }^{175}$ M. Zeman, ${ }^{125}$ A. Zemla, ${ }^{39}$ C. Zendler,${ }^{21}$ O. Zenin, ${ }^{128}$ T. Ženiš, ${ }^{144 a}$ Z. Zinonos, ${ }^{122 a, 122 b}$ S. Zenz, ${ }^{15}$ D. Zerwas, ${ }^{115}$ G. Zevi della Porta ${ }^{57}$ Z. Zhan, ${ }^{33 d}$ D. Zhang, ${ }^{33 b, 11}$ H. Zhang, ${ }^{88}$ J. Zhang, ${ }^{6}$ X. Zhang, ${ }^{33 d}$ Z. Zhang, ${ }^{115}$ L. Zhao, ${ }^{108}$ T. Zhao ${ }^{138}$ Z. Zhao, ${ }^{33 b}$ A. Zhemchugov, ${ }^{64}$ J. Zhong, ${ }^{118}$ B. Zhou, ${ }^{87}$ N. Zhou, ${ }^{163}$ Y. Zhou, ${ }^{151}$ C. G. Zhu, ${ }^{33 d}$ H. Zhu, ${ }^{42}$ J. Zhu, ${ }^{87}$ Y. Zhu, ${ }^{33 b}$ X. Zhuang, ${ }^{98}$ V. Zhuravlov, ${ }^{99}$ D. Zieminska, ${ }^{60}$ N. I. Zimin, ${ }^{64}$ R. Zimmermann, ${ }^{21}$ S. Zimmermann, ${ }^{21}$ S. Zimmermann, ${ }^{48}$ M. Ziolkowski, ${ }^{141}$ R. Zitoun, ${ }^{5}$ L. Živković, ${ }^{35}$ V. V. Zmouchko, ${ }^{128, a}$ G. Zobernig, ${ }^{173}$ A. Zoccoli, ${ }^{20 a, 20 b}$ M. zur Nedden, ${ }^{16}$ V. Zutshi, ${ }^{106}$ and L. Zwalinski ${ }^{30}$

(ATLAS Collaboration)

\footnotetext{
${ }^{1}$ School of Chemistry and Physics, University of Adelaide, North Terrace Campus, 5000, SA, Australia

${ }^{2}$ Physics Department, SUNY Albany, Albany New York, USA

${ }^{3}$ Department of Physics, University of Alberta, Edmonton Alberta, Canada

${ }^{4 a}$ Department of Physics, Ankara University, Ankara, Turkey

${ }^{4 \mathrm{~b}}$ Department of Physics, Dumlupinar University, Kutahya, Turkey

${ }^{4 \mathrm{c}}$ Department of Physics, Gazi University, Ankara, Turkey

${ }^{4 \mathrm{~d} D i v i s i o n}$ of Physics, TOBB University of Economics and Technology, Ankara, Turkey ${ }^{4 \mathrm{e}}$ Turkish Atomic Energy Authority, Ankara, Turkey

${ }^{5}$ LAPP, CNRS/IN2P3 and Université de Savoie, Annecy-le-Vieux, France

${ }^{6}$ High Energy Physics Division, Argonne National Laboratory, Argonne Illinois, USA

${ }^{7}$ Department of Physics, University of Arizona, Tucson Arizona, USA

${ }^{8}$ Department of Physics, The University of Texas at Arlington, Arlington Texas, USA

${ }^{9}$ Physics Department, University of Athens, Athens, Greece

${ }^{10}$ Physics Department, National Technical University of Athens, Zografou, Greece

${ }^{11}$ Institute of Physics, Azerbaijan Academy of Sciences, Baku, Azerbaijan

${ }^{12}$ Institut de Física d'Altes Energies and Departament de Física de la Universitat Autònoma de Barcelona and ICREA, Barcelona, Spain

${ }^{13 a}$ Institute of Physics, University of Belgrade, Belgrade, Serbia

${ }^{13 \mathrm{~b}}$ Vinca Institute of Nuclear Sciences, University of Belgrade, Belgrade, Serbia

${ }^{14}$ Department for Physics and Technology, University of Bergen, Bergen, Norway

${ }^{15}$ Physics Division, Lawrence Berkeley National Laboratory and University of California, Berkeley California, USA

${ }^{16}$ Department of Physics, Humboldt University, Berlin, Germany

${ }^{17}$ Albert Einstein Center for Fundamental Physics and Laboratory for High Energy Physics, University of Bern, Bern, Switzerland

${ }^{18}$ School of Physics and Astronomy, University of Birmingham, Birmingham, United Kingdom

${ }^{19 a}$ Department of Physics, Bogazici University, Istanbul, Turkey

${ }^{19 \mathrm{~b}}$ Division of Physics, Dogus University, Istanbul, Turkey

${ }^{19 \mathrm{c}}$ Department of Physics Engineering, Gaziantep University, Gaziantep, Turkey

${ }^{19 \mathrm{~d}}$ Department of Physics, Istanbul Technical University, Istanbul, Turkey

${ }^{20 \mathrm{a}}$ INFN Sezione di Bologna, Italy

${ }^{20 \mathrm{~b}}$ Dipartimento di Fisica, Università di Bologna, Bologna, Italy

${ }^{21}$ Physikalisches Institut, University of Bonn, Bonn, Germany

${ }^{22}$ Department of Physics, Boston University, Boston Massachusetts, USA

${ }^{23}$ Department of Physics, Brandeis University, Waltham Massachusetts, USA

${ }^{24 a}$ Universidade Federal do Rio De Janeiro COPPE/EE/IF, Rio de Janeiro, Brazil

${ }^{24 \mathrm{~b}}$ Federal University of Juiz de Fora (UFJF), Juiz de Fora, Brazil
} 
${ }^{24 \mathrm{c}}$ Federal University of Sao Joao del Rei (UFSJ), Sao Joao del Rei, Brazil

${ }^{24 \mathrm{~d}}$ Instituto de Fisica, Universidade de Sao Paulo, Sao Paulo, Brazil

${ }^{25}$ Physics Department, Brookhaven National Laboratory, Upton New York, USA

${ }^{26 a}$ National Institute of Physics and Nuclear Engineering, Bucharest, Romania

${ }^{26 \mathrm{~b}}$ University Politehnica Bucharest, Bucharest, Romania

${ }^{26 c}$ West University in Timisoara, Timisoara, Romania

${ }^{27}$ Departamento de Física, Universidad de Buenos Aires, Buenos Aires, Argentina

${ }^{28}$ Cavendish Laboratory, University of Cambridge, Cambridge, United Kingdom

${ }^{29}$ Department of Physics, Carleton University, Ottawa Ontario, Canada

${ }^{30}$ CERN, Geneva, Switzerland

${ }^{31}$ Enrico Fermi Institute, University of Chicago, Chicago Illinois, USA

${ }^{32 a}$ Departamento de Física, Pontificia Universidad Católica de Chile, Santiago, Chile

${ }^{32 \mathrm{~b}}$ Departamento de Física, Universidad Técnica Federico Santa María, Valparaíso, Chile

${ }^{33 a}$ Institute of High Energy Physics, Chinese Academy of Sciences, Beijing, China

${ }^{33 \mathrm{~b}}$ Department of Modern Physics, University of Science and Technology of China, Anhui, China

${ }^{33 \mathrm{c}}$ Department of Physics, Nanjing University, Jiangsu, China

${ }^{33 d}$ School of Physics, Shandong University, Shandong, China

${ }^{34}$ Laboratoire de Physique Corpusculaire, Clermont Université and Université Blaise Pascal and CNRS/IN2P3,

Aubiere Cedex, France

${ }^{35}$ Nevis Laboratory, Columbia University, Irvington New York, USA

${ }^{36}$ Niels Bohr Institute, University of Copenhagen, Kobenhavn, Denmark

${ }^{37 a}$ INFN Gruppo Collegato di Cosenza, Italy

${ }^{37 \mathrm{~b}}$ Dipartimento di Fisica, Università della Calabria, Arcavata di Rende, Italy

${ }^{38}$ AGH University of Science and Technology, Faculty of Physics and Applied Computer Science, Krakow, Poland

${ }^{39}$ The Henryk Niewodniczanski Institute of Nuclear Physics, Polish Academy of Sciences, Krakow, Poland

${ }^{40}$ Physics Department, Southern Methodist University, Dallas Texas, USA

${ }^{41}$ Physics Department, University of Texas at Dallas, Richardson Texas, USA

${ }^{42}$ DESY, Hamburg and Zeuthen, Germany

${ }^{43}$ Institut für Experimentelle Physik IV, Technische Universität Dortmund, Dortmund, Germany

${ }^{44}$ Institut für Kern- und Teilchenphysik, Technical University Dresden, Dresden, Germany

${ }^{45}$ Department of Physics, Duke University, Durham North Carolina, USA

${ }^{46}$ SUPA-School of Physics and Astronomy, University of Edinburgh, Edinburgh, United Kingdom

${ }^{47}$ INFN Laboratori Nazionali di Frascati, Frascati, Italy

${ }^{48}$ Fakultät für Mathematik und Physik, Albert-Ludwigs-Universität, Freiburg, Germany

${ }^{49}$ Section de Physique, Université de Genève, Geneva, Switzerland

${ }^{50 a}$ INFN Sezione di Genova, Italy

${ }^{50 \mathrm{~b}}$ Dipartimento di Fisica, Università di Genova, Genova, Italy

${ }^{51 a}$ E. Andronikashvili Institute of Physics, Tbilisi State University, Tbilisi, Georgia

${ }^{51 \mathrm{~b}}$ High Energy Physics Institute, Tbilisi State University, Tbilisi, Georgia

${ }^{52}$ II Physikalisches Institut, Justus-Liebig-Universität Giessen, Giessen, Germany

${ }^{53}$ SUPA-School of Physics and Astronomy, University of Glasgow, Glasgow, United Kingdom

${ }^{54}$ II Physikalisches Institut, Georg-August-Universität, Göttingen, Germany

${ }^{55}$ Laboratoire de Physique Subatomique et de Cosmologie, Université Joseph Fourier and CNRS/IN2P3 and Institut National Polytechnique de Grenoble, Grenoble, France

${ }^{56}$ Department of Physics, Hampton University, Hampton Virginia, USA

${ }^{57}$ Laboratory for Particle Physics and Cosmology, Harvard University, Cambridge Massachusetts, USA

${ }^{58}$ Kirchhoff-Institut für Physik, Ruprecht-Karls-Universität Heidelberg, Heidelberg, Germany

${ }^{58 \mathrm{~b}}$ Physikalisches Institut, Ruprecht-Karls-Universität Heidelberg, Heidelberg, Germany

${ }^{58 \mathrm{c}}$ ZITI Institut für technische Informatik, Ruprecht-Karls-Universität Heidelberg, Mannheim, Germany

${ }^{59}$ Faculty of Applied Information Science, Hiroshima Institute of Technology, Hiroshima, Japan

${ }^{60}$ Department of Physics, Indiana University, Bloomington Indiana, USA

${ }^{61}$ Institut für Astro- und Teilchenphysik, Leopold-Franzens-Universität, Innsbruck, Austria

${ }^{62}$ University of Iowa, Iowa City Iowa, USA

${ }^{63}$ Department of Physics and Astronomy, Iowa State University, Ames Iowa, USA

${ }^{64}$ Joint Institute for Nuclear Research, JINR Dubna, Dubna, Russia

${ }^{65}$ KEK, High Energy Accelerator Research Organization, Tsukuba, Japan

${ }^{66}$ Graduate School of Science, Kobe University, Kobe, Japan

${ }^{67}$ Faculty of Science, Kyoto University, Kyoto, Japan

${ }^{68}$ Kyoto University of Education, Kyoto, Japan

${ }^{69}$ Department of Physics, Kyushu University, Fukuoka, Japan

${ }^{70}$ Instituto de Física La Plata, Universidad Nacional de La Plata and CONICET, La Plata, Argentina 


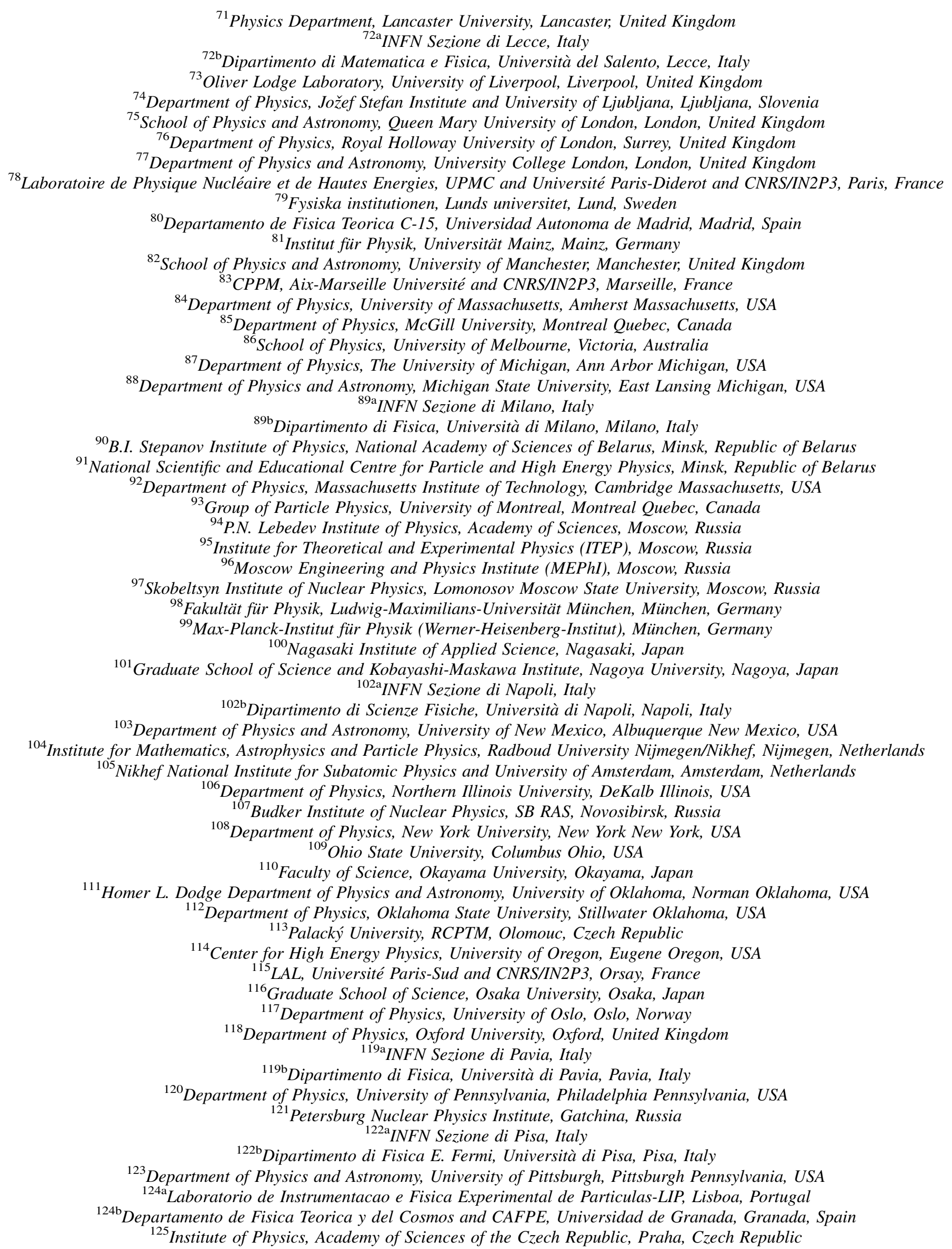


${ }^{126}$ Faculty of Mathematics and Physics, Charles University in Prague, Praha, Czech Republic

${ }^{127}$ Czech Technical University in Prague, Praha, Czech Republic

${ }^{128}$ State Research Center Institute for High Energy Physics, Protvino, Russia

${ }^{129}$ Particle Physics Department, Rutherford Appleton Laboratory, Didcot, United Kingdom

${ }^{130}$ Physics Department, University of Regina, Regina Saskatchewan, Canada

${ }^{131}$ Ritsumeikan University, Kusatsu, Shiga, Japan

${ }^{132 a}$ INFN Sezione di Roma I, Italy

${ }^{132 b}$ Dipartimento di Fisica, Università La Sapienza, Roma, Italy

${ }^{133 a}$ INFN Sezione di Roma Tor Vergata, Italy

${ }^{133 b}$ Dipartimento di Fisica, Università di Roma Tor Vergata, Roma, Italy

${ }^{134 a}$ INFN Sezione di Roma Tre, Italy

${ }^{134 \mathrm{~b}}$ Dipartimento di Fisica, Università Roma Tre, Roma, Italy

${ }^{135 a}$ Faculté des Sciences Ain Chock, Réseau Universitaire de Physique des Hautes Energies-Université Hassan II, Casablanca, Morocco

${ }^{135 \mathrm{~b}}$ Centre National de l'Energie des Sciences Techniques Nucleaires, Rabat, Morocco

${ }^{135 c}$ Faculté des Sciences Semlalia, Université Cadi Ayyad, LPHEA-Marrakech, Morocco

${ }^{135 \mathrm{~d}}$ Faculté des Sciences, Université Mohamed Premier and LPTPM, Oujda, Morocco

${ }^{135 \mathrm{e}}$ Faculté des sciences, Université Mohammed V-Agdal, Rabat, Morocco

${ }^{136}$ DSM/IRFU (Institut de Recherches sur les Lois Fondamentales de l'Univers),

CEA Saclay (Commissariat a l'Energie Atomique), Gif-sur-Yvette, France

${ }^{137}$ Santa Cruz, Institute for Particle Physics, University of California Santa Cruz, Santa Cruz California, USA

${ }^{138}$ Department of Physics, University of Washington, Seattle Washington, USA

${ }^{139}$ Department of Physics and Astronomy, University of Sheffield, Sheffield, United Kingdom

${ }^{140}$ Department of Physics, Shinshu University, Nagano, Japan

${ }^{141}$ Fachbereich Physik, Universität Siegen, Siegen, Germany

${ }^{142}$ Department of Physics, Simon Fraser University, Burnaby British Columbia, Canada

${ }^{143}$ SLAC National Accelerator Laboratory, Stanford California, USA

${ }^{144 a}$ Faculty of Mathematics, Physics \& Informatics, Comenius University, Bratislava, Slovak Republic

${ }^{144 b}$ Department of Subnuclear Physics, Institute of Experimental Physics of the Slovak Academy of Sciences, Kosice, Slovak Republic

${ }^{145 a}$ Department of Physics, University of Johannesburg, Johannesburg, South Africa

${ }^{145 \mathrm{~b}}$ School of Physics, University of the Witwatersrand, Johannesburg, South Africa

${ }^{146 a}$ Department of Physics, Stockholm University, Sweden

${ }^{146 \mathrm{~b}}$ The Oskar Klein Centre, Stockholm, Sweden

${ }^{147}$ Physics Department, Royal Institute of Technology, Stockholm, Sweden

${ }^{148}$ Departments of Physics \& Astronomy and Chemistry, Stony Brook University, Stony Brook New York, USA

${ }^{149}$ Department of Physics and Astronomy, University of Sussex, Brighton, United Kingdom

${ }^{150}$ School of Physics, University of Sydney, Sydney, Australia

${ }^{151}$ Institute of Physics, Academia Sinica, Taipei, Taiwan

${ }^{152}$ Department of Physics, Technion: Israel Institute of Technology, Haifa, Israel

${ }^{153}$ Raymond and Beverly Sackler School of Physics and Astronomy, Tel Aviv University, Tel Aviv, Israel

${ }^{154}$ Department of Physics, Aristotle University of Thessaloniki, Thessaloniki, Greece

${ }^{155}$ International Center for Elementary Particle Physics and Department of Physics, The University of Tokyo, Tokyo, Japan

${ }^{156}$ Graduate School of Science and Technology, Tokyo Metropolitan University, Tokyo, Japan

${ }^{157}$ Department of Physics, Tokyo Institute of Technology, Tokyo, Japan

${ }^{158}$ Department of Physics, University of Toronto, Toronto Ontario, Canada

${ }^{159 a}$ TRIUMF, Vancouver British Columbia, Canada

${ }^{159 \mathrm{~b}}$ Department of Physics and Astronomy, York University, Toronto Ontario, Canada

${ }^{160}$ Institute of Pure and Applied Sciences, University of Tsukuba,1-1-1 Tennodai, Tsukuba, Ibaraki 305-8571, Japan

${ }^{161}$ Science and Technology Center, Tufts University, Medford Massachusetts, USA

${ }^{162}$ Centro de Investigaciones, Universidad Antonio Narino, Bogota, Colombia

${ }^{163}$ Department of Physics and Astronomy, University of California Irvine, Irvine California, USA

${ }^{164 a}$ INFN Gruppo Collegato di Udine, Italy ${ }^{164 \mathrm{~b}}$ ICTP, Trieste, Italy

${ }^{164 \mathrm{c}}$ Dipartimento di Chimica, Fisica e Ambiente, Università di Udine, Udine, Italy

${ }^{165}$ Department of Physics, University of Illinois, Urbana Illinois, USA

${ }^{166}$ Department of Physics and Astronomy, University of Uppsala, Uppsala, Sweden

${ }^{167}$ Instituto de Física Corpuscular (IFIC) and Departamento de Física Atómica, Molecular y Nuclear and Departamento de Ingeniería Electrónica and Instituto de Microelectrónica de Barcelona (IMB-CNM), University of Valencia and CSIC, Valencia, Spain

${ }^{168}$ Department of Physics, University of British Columbia, Vancouver British Columbia, Canada

${ }^{169}$ Department of Physics and Astronomy, University of Victoria, Victoria British Columbia, Canada

${ }^{170}$ Department of Physics, University of Warwick, Coventry, United Kingdom 


\author{
${ }^{171}$ Waseda University, Tokyo, Japan \\ ${ }^{172}$ Department of Particle Physics, The Weizmann Institute of Science, Rehovot, Israel \\ ${ }^{173}$ Department of Physics, University of Wisconsin, Madison Wisconsin, USA \\ ${ }^{174}$ Fakultät für Physik und Astronomie, Julius-Maximilians-Universität, Würzburg, Germany \\ ${ }^{175}$ Fachbereich C Physik, Bergische Universität Wuppertal, Wuppertal, Germany \\ ${ }^{176}$ Department of Physics, Yale University, New Haven Connecticut, USA \\ ${ }^{177}$ Yerevan Physics Institute, Yerevan, Armenia \\ ${ }^{178}$ Domaine scientifique de la Doua, Centre de Calcul CNRS/IN2P3, Villeurbanne Cedex, France
}

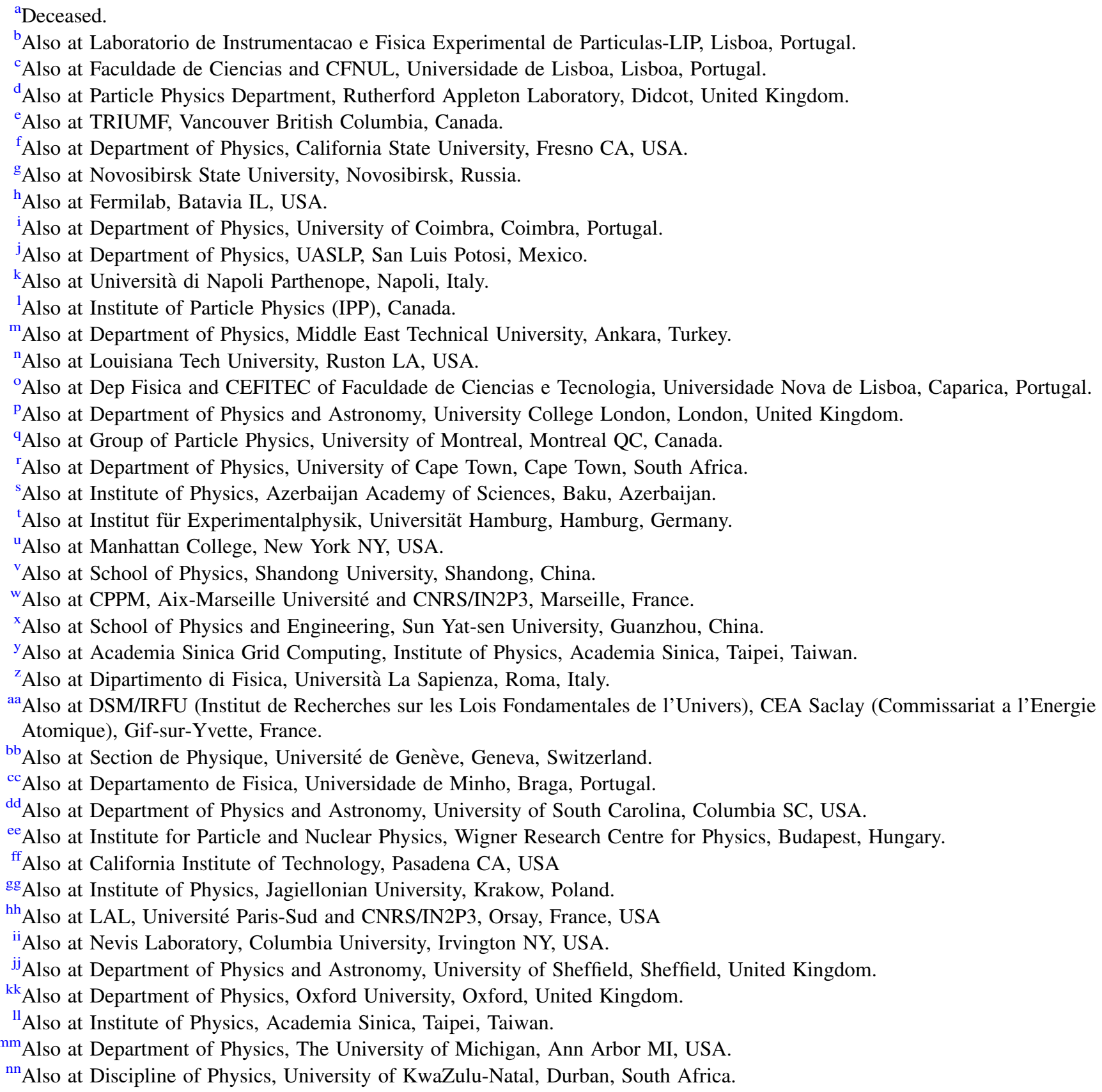

